\title{
Phase noise analysis of clock recovery based on an optoelectronic phase-locked loop
}

\author{
Zibar, Darko; Mørk, Jesper; Oxenløwe, Leif Katsuo; Clausen, Anders
}

Published in:

Journal of Lightwave Technology

Link to article, DOI:

10.1109/JLT.2006.890433

Publication date:

2007

Document Version

Publisher's PDF, also known as Version of record

Link back to DTU Orbit

Citation (APA):

Zibar, D., Mørk, J., Oxenløwe, L. K., \& Clausen, A. (2007). Phase noise analysis of clock recovery based on an optoelectronic phase-locked loop. Journal of Lightwave Technology, 25(3), 901-914.

https://doi.org/10.1109/JLT.2006.890433

\section{General rights}

Copyright and moral rights for the publications made accessible in the public portal are retained by the authors and/or other copyright owners and it is a condition of accessing publications that users recognise and abide by the legal requirements associated with these rights.

- Users may download and print one copy of any publication from the public portal for the purpose of private study or research.

- You may not further distribute the material or use it for any profit-making activity or commercial gain

- You may freely distribute the URL identifying the publication in the public portal 


\title{
Phase Noise Analysis of Clock Recovery Based on an Optoelectronic Phase-Locked Loop
}

\author{
Darko Zibar, Member, IEEE, Jesper Mørk, Leif Katsuo Oxenløwe, and Anders T. Clausen
}

\begin{abstract}
A detailed theoretical analysis of a clock-recovery (CR) scheme based on an optoelectronic phase-locked loop is presented. The analysis emphasizes the phase noise performance, taking into account the noise of the input data signal, the local voltage-controlled oscillator (VCO), and the laser employed in the loop. The effects of loop time delay and the laser transfer function are included in the stochastic differential equations describing the system, and a detailed timing jitter analysis of this type of optoelectronic CR for high-speed optical-time-division-multiplexing systems is performed. It is shown that a large loop length results in a higher timing jitter of the recovered clock signal. The impact of the loop length on the clock signal jitter can be reduced by using a low-noise VCO and a low loop filter bandwidth. Using the model, the timing jitter of the recovered optical and electrical clock signal can be evaluated. We numerically investigate the timing jitter requirements for combined electrical/optical local oscillators, in order for the recovered clock signal to have less jitter than that of the input signal. The timing jitter requirements for the free-running laser and the VCO are more relaxed for the extracted optical clock (lasers's output) signal.
\end{abstract}

Index Terms-Clock recovery (CR), optical time division multiplexing (OTDM), optoelectronic phase-locked loop (OPLL), oscillator noise, phase noise, stochastic differential equations, time delay, timing jitter.

\section{INTRODUCTION}

$\mathbf{P}$ HASE-LOCKED LOOPS (PLLs) are used in many applications involving phase and frequency synchronization in control systems [1]-[5], clock generation in digital signal processors [6]-[8], clock extraction in high-speed optical communication systems [9]-[16], and, recently, high-frequency low-noise signal generation [17]-[20]. The realization of the PLL depends on the application, and therefore, various PLL configurations exist. In this paper, we focus on a balanced optoelectronic PLL (OPLL) used for clock extraction from highspeed optical time division multiplexed (OTDM) signals and analyze its stability and noise properties. The block diagram of the balanced OPLL is shown in Fig. 1. The phase comparator (PC) mixes the optical high-speed data signal (e.g., $160 \mathrm{~Gb} / \mathrm{s}$ ) with a locally generated optical clock signal at the base rate frequency (e.g., $10 \mathrm{GHz}$ ), producing an error signal. The mixing

Manuscript received January 3, 2006; revised December 4, 2006. This work was supported by the Danish Research Council under Project Ultra-Net.

The authors are with Research Center COM, Technical University of Denmark, 2800 Lyngby, Denmark (e-mail: dz@com.dtu.dk; jm@com.dtu.dk; lo@com.dtu.dk; atc@com.dtu.dk).

Color versions of one or more of the figures in this paper are available online at http://ieeexplore.ieee.org.

Digital Object Identifier 10.1109/JLT.2006.890433

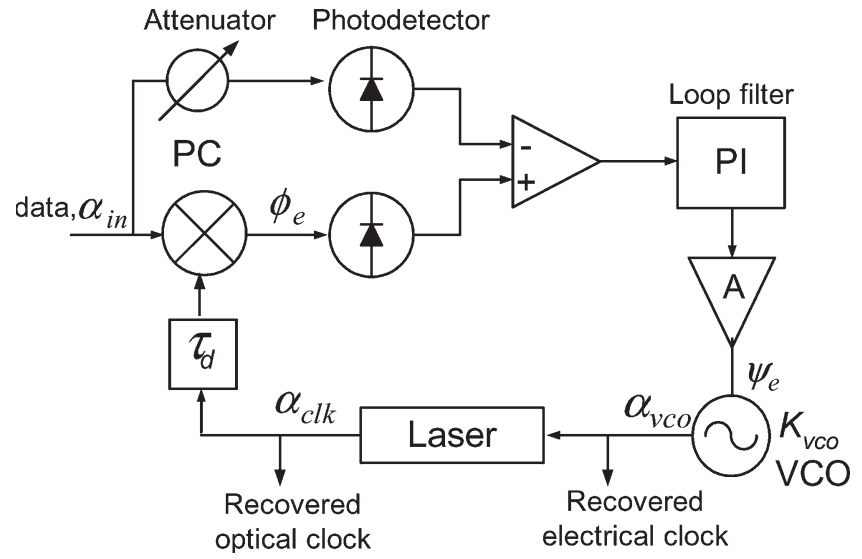

Fig. 1. Diagram of the balanced OPLL-based CR. $\tau_{\mathrm{d}}$ : the total time delay in the loop, PC: phase comparator, PI: proportional integrator, $A$ : amplifier gain, VCO: voltage-controlled oscillator, $K_{\mathrm{vco}}$ : gain of the voltage-controlled oscillator, $\alpha_{\mathrm{in}}$ : input phase noise, $\alpha_{\mathrm{clk}}$ : phase noise of the laser, $\alpha_{\mathrm{vco}}$ : phase noise of the VCO, $\phi_{e}$ : phase difference between $\alpha_{\mathrm{clk}}$ and $\alpha_{\mathrm{in}}$, and $\psi_{e}$ : applied signal to the $\mathrm{VCO}$.

process corresponds to a mathematical multiplication with a mixer gain $\mathrm{G}$. The mixer output is a slowly varying signal proportional to the sine of the phase difference between the data and the optical clock signal. In practice, nonlinear processes like four wave mixing in a semiconductor optical amplifier can be used to achieve the mixing [9]. The optical error signal is then converted into the electrical domain.

The balanced (low bandwidth) photodetection provides the subtraction of the de level from the error signal, which results in a bipolar error signal. This subtraction also helps to stabilize the error signal against fluctuations in the input signal power levels. The signal is then low pass filtered and fed back to the voltagecontrolled oscillator (VCO), which controls the frequency of the optical clock generating laser. In an OPLL-based clock recovery (CR), a considerable time delay is very likely to occur, since the loop length of an OPLL is often much longer than in an electrical PLL. Time delays in an OPLL may typically arise from the presence of a fiber amplifier [erbium-doped fiber amplifiers (EDFA)] and/or a pulse compression stage in the loop. An EDFA may also sometimes be needed to amplify the optical clock signal before the PC. It has been demonstrated that intraloop time delays destabilize the loop [21], [22]. Theoretical investigations and modeling of the PLLs, including noise, is important in order to understand the limitations and improve the properties of circuits based on phase-locking. A large amount of literature is available on this topic; see [2] and [7]. Possibly, the most general and rigorous treatment of the topic is that of Mehrotra [7]. However, compared to electrical PLLs, the 
loop length of an OPLL is longer, and therefore, the time delay must be included in the model. Furthermore, in an OPLL, there are two oscillators, an electrical VCO, and an optical clock generating laser, and the timing jitter of the extracted clock signal will thereby be influenced by the phase noise of both oscillators. The phase noise of the recovered clock signal is filtered by the laser transfer function, with a characteristic knee frequency $f_{\text {ch }}$, and thus, this feature must also be included in the model equations.

In this paper, a detailed phase noise analysis of an OPLL is performed. A set of stochastic differential (Langevin) equations describing the total phase error in the OPLL is derived. By a small-signal analysis, the Langevin equations are linearized, and the associated system of stochastic differential equations is solved using Fourier techniques, and we derive an analytical expressions for the correlation functions and probability density function (PDF) of the recovered clock signal. Using the model, the timing jitter of the recovered optical and electrical clock signals are calculated and compared.

This paper is organized as follows. In Sections II and III, the Langevin equations describing the OPLL are derived. In Section IV, we use the Fourier transformation technique to obtain the correlation functions, which are used in Section V to compute the autocorrelation function of the extracted clock signal. In Section VI, we derive a pdf for the recovered clock signal. Finally, in Section VII, we investigate how the loop gain, the loop filter bandwidth, the time delay, and the laser characteristic knee frequency influence the timing jitter of the recovered clock signal. By using the model, timing jitter requirements for the electrical and optical clock sources are determined in order to obtain a recovered clock signal with less timing jitter than the degraded input data signal. Also, we show that this is achievable, as long as the jitter of the free-running VCO is less than the input signal jitter, even though the free-running optical clock signal generating laser contains more timing jitter than the input. This result may relax timing jitter requirements when designing optical pulse sources for high-bit-rate systems.

\section{Derivation of LANGevin Equations}

In order to study the dynamical behavior of the OPLL (see Fig. 1), stochastic differential equations describing the phase error between input signal and local oscillators need to be derived. The intensity of the input signal in the presence of phase noise $\alpha_{\text {in }}(t)$ originating from the pulse source at the transmitter, regenerators, and in-line amplifiers is expressed in Fourier series as [23]

$$
P_{\text {in }}\left(t+\alpha_{\text {in }}(t)\right)=s_{0}+2 \sum_{k=1}^{\infty} s_{k} \sin \left[2 \pi k f_{0} t+2 \pi k f_{0} \alpha_{\text {in }}(t)\right]
$$

where $k$ is a positive integer. The constants $s_{0}$ and $s_{k}$ are Fourier coefficients, and $f_{0}$ is the frequency of the aggregate bit rate (e.g., 40, 160, $320 \mathrm{GHz}$, etc.) [24]. The input data signal phase noise $\alpha_{\text {in }}(t)$ is expressed as $\alpha_{\text {in }}(t)=\sqrt{c_{\text {in }}} B_{\text {in }}(t)$ [25], where $B_{\text {in }}(t)$ is described by a 1-D Brownian motion stochastic process, and $c_{\mathrm{in}}$ is a constant determining the amount of phase noise associated with the input signal. For a more detailed explanation, see the Appendix. The intensity of a locally generated optical clock signal is similarly expressed by a Fourier series as [23]

$$
\begin{aligned}
& P_{\mathrm{clk}}\left(t+\alpha_{\mathrm{clk}}(t)\right) \\
& =c_{0}+2 \sum_{q=1}^{\infty} c_{q} \cos \left[2 \pi q\left(f_{0}^{\prime} / m\right) t+2 \pi q\left(f_{0}^{\prime} / m\right) \alpha_{\mathrm{clk}}(t)\right]
\end{aligned}
$$

where $q$ is a positive integer. The constants $c_{0}$ and $c_{q}$ are Fourier coefficients. The repetition frequency of the optical clock signal is $f_{0}^{\prime} / m$, where $m$ is an integer. The frequency $f_{0}^{\prime} / m$ (e.g., 10 or $40 \mathrm{GHz}$ ) corresponds to the free-running frequency of the optical clock signal generating pulse source, and it is chosen so that it is close to the base rate frequency $f_{\text {base }}$ (e.g., $10 \mathrm{GHz}$, $40 \mathrm{GHz}$ ) of the optical input data signal [24]. $\alpha_{\mathrm{clk}}(t)$ is the phase noise of the optical clock signal and is modeled as a bandlimited Brownian motion stochastic process, as later shown in (9). The output of the PC (mixer) (see Fig. 1) is defined to be a product between the optical clock signal in (2) and the optical data signal in (1) with a gain $G$. PCs are frequently modeled as multipliers, partly for analytical convenience and partly because many practical PCs are good approximations to multipliers [2]. The optical signal after the mixing contains low-frequency components as well as high-frequency components, which exceed the aggregate bit rate $\left(>f_{0}\right)$. A slow photodiode with bandwidth $\mathrm{BW} \ll f_{\text {base }}$ is used to convert the signal from the optical to the electrical domain and is also used to filter out high-frequency components. It is assumed that the responsivity of the photodiode is constant in frequency. The fundamental frequency component of the optical data signal $f_{0}(k=1)$ interacts with the $m$ th frequency component $(q=m)$ of the optical clock signal, resulting in the frequency component $\Delta f=\left(f_{0}^{\prime}-f_{0}\right)$. When the aggregate bit rate of the optical data signal is $160 \mathrm{~Gb} / \mathrm{s}$ and the base rate is $10 \mathrm{~Gb} / \mathrm{s}$, we have $m=16$. The frequency component $\Delta f$ is smaller than the bandwidth of the photodiode and can therefore be detected and forms the fundamental frequency component. Furthermore, following Fig. 1, it can be observed that part of the optical data signal is first attenuated, in order to match the signal powers, and then passed through the second photodiode, which is identical to first one. Since the photodiode is slow, only the dc frequency component of the optical data signal passes through. The balanced photodetection (BW in the megahertz range) thereby provides a subtraction of the dc level from the error signal, and the error signal after balanced photodetection is expressed as

$$
\begin{gathered}
e(t)=R G s_{0}^{\prime} c_{0}^{\prime}+2 R G \sum_{i=1}^{\infty} s_{i} c_{i \cdot m} \sin \left[i \Phi_{e}(t)\right] \\
\Phi_{e}(t)=\Delta \omega t+2 \pi f_{0}^{\prime} \alpha_{\mathrm{clk}}(t)-2 \pi f_{0} \alpha_{\mathrm{in}}(t)
\end{gathered}
$$

where $i$ is a positive integer, and $R$ is the responsivity of the photodiode. $s_{0}^{\prime} c_{0}^{\prime}$ is the remaining dc level in the error signal after the subtraction. Even though the balanced photodetection provides a subtraction of the dc levels, cancellation may not be complete due to imperfections in the electronics. 
In order to perform phase noise analysis of the system, the locally generated optical clock signal must be synchronized to the input data signal, i.e., $f_{0}=f_{0}^{\prime}$. We choose to operate with a normalized total phase difference $\phi_{e}(t) \equiv \Phi_{e}(t) /\left(2 \pi f_{0}\right)$. The advantage of using the normalized form of $\phi_{e}(t)$ is that the phase noise analysis will not be affected by the frequency difference between the input signal and the locally generated clock signal. The normalized phase difference $\phi_{e}(t)$ is given by

$$
\phi_{e}(t)=\Delta \omega_{\mathrm{n}} t+\alpha_{\mathrm{clk}}(t)-\alpha_{\mathrm{in}}(t)
$$

where $\Delta \omega_{\mathrm{n}}=\Delta \omega /\left(2 \pi f_{0}\right)$. The normalized error signal $e_{\mathrm{n}}(t) \equiv R G s_{0}^{\prime} c_{0}^{\prime}+2 R G \sum_{i=1}^{\infty} s_{i} c_{i \cdot m} \sin \left[i \phi_{e}(t)\right]$ is now applied to the loop filter: a Proportional Integrator (PI). In the time domain the output of the PI filter, $\psi_{e}(t)$ is defined as [3]

$$
\tau_{1} \frac{d \psi_{e}}{d t}=A\left[e_{\mathrm{n}}(t)-\tau_{2} \frac{d e_{\mathrm{n}}}{d t}\right]
$$

where $A$ is the gain of the electrical amplifier in the loop. $\tau_{1}$ represents the integration time of the filter (inversely proportional to the PI filter bandwidth). Furthermore, dc gain of the PI filter scales linearly with $\tau_{2}$. The reason that we concentrate on a PI loop filter is because it is more tolerant to long loop lengths compared to the low-pass and active-lag filters [22]. The output signal of the VCO is written as

$$
v(t)=V_{0} \cos \left[2 \pi f_{0}^{\prime \prime} t+2 \pi f_{0}^{\prime \prime} \alpha_{\mathrm{vco}}(t)\right]
$$

where $f_{0}^{\prime \prime}$ is the free-running frequency of the $\mathrm{VCO}$, which is also chosen close to the base rate of the optical data signal, and $V_{0}$ is the amplitude of the VCO signal. $\alpha_{\mathrm{vco}}(t)$ is the phase noise associated with the VCO. It has been shown by Mehrotra [7] that the phase noise of the oscillator in the presence of the applied signal is governed by the following stochastic differential equation:

$$
\frac{d \alpha_{\mathrm{vco}}}{d t}=\nu^{\mathrm{T}}\left(t+\alpha_{\mathrm{vco}}(t)\right) \Gamma_{p}(t)+\nu_{\mathrm{ctrl}}\left(t+\alpha_{\mathrm{vco}}(t)\right) \psi_{e}(t)
$$

where $\Gamma_{p}(t)$ is a vector of $p$ uncorrelated white noise sources, and $\nu(\cdot)$ is a periodic function which depends on the noise source intensities and the response of the linearized oscillator circuit. $\nu_{\text {ctrl }}(\cdot)$ is the component of $\nu(\cdot)$ which corresponds to a unit noise source present at the control node of the VCO. In order to perform noise analysis of the PLL, we must assume that the loop is locked and that we are therefore only interested in the asymptotic behavior of the VCO phase noise. Asymptotically, (7) reduces to [7]

$$
\frac{d \alpha_{\mathrm{vco}}}{d t}=\sqrt{c_{\mathrm{vco}}} \Gamma_{\mathrm{vco}}(t)+K_{\mathrm{vco}} \psi_{e}(t)
$$

where $c_{\mathrm{vco}}$ is a constant determining the amount of phase noise associated with the free-running $\mathrm{VCO}$, and $K_{\mathrm{vco}}$ is the average gain of the VCO. $\Gamma_{\text {vco }}(t)$ is a white noise source. From the diagram of the OPLL, as shown in Fig. 1, we observe that the signal from the VCO is directly modulating the optical pulse source (laser). Under the assumption that the VCO and laser are synchronized, the phase noise of the $\operatorname{VCO} \alpha_{\mathrm{vco}}(t)$ will be filtered by the laser transfer function, with a characteristic knee frequency of $f_{\mathrm{ch}}$ [27], [28]. The characteristic knee frequency $f_{\text {ch }}$ denotes the bandwidth around the laser's center frequency within which phase noise is transferred from the VCO to the laser [29]. The phase noise $\alpha_{\mathrm{clk}}(t)$ of the laser is expressed as

$$
\frac{d \alpha_{\mathrm{clk}}}{d t}=-\frac{\gamma_{e}(t)}{\tau_{\mathrm{ch}}}+\sqrt{c_{\mathrm{clk}}} \Gamma_{\mathrm{clk}}(t)
$$

where $\tau_{\mathrm{ch}}=1 /\left(2 \pi f_{\mathrm{ch}}\right)$, and $c_{\mathrm{clk}}$ is a constant describing the magnitude of the phase noise of the free-running laser described by the white noise source $\Gamma_{\text {clk }}(t) . \gamma_{e}(t)$ is the phase noise difference between the VCO and the optical pulse source, i.e., $\gamma_{e}(t)=\alpha_{\mathrm{clk}}(t)-\alpha_{\mathrm{vco}}(t)$. The stochastic differential equation describing the phase noise difference between the VCO and the optical pulse source is then obtained by differentiating $\gamma_{e}(t)$ and using (8) and (9):

$$
\frac{d \gamma_{e}}{d t}=-\frac{\gamma_{e}(t)}{\tau_{\mathrm{ch}}}-K_{\mathrm{vco}} \psi_{e}(t)+\sqrt{c_{\mathrm{clk}}} \Gamma_{\mathrm{clk}}(t)-\sqrt{c_{\mathrm{vco}}} \Gamma_{\mathrm{vco}}(t) .
$$

The effect of a time delay $\tau_{\mathrm{d}}$ is taken into account by incorporating a delay in $\alpha_{\mathrm{clk}}(t)$, (4) (see Fig. 1). Therefore, $\phi_{e}(t)$ in (4) changes to $\phi_{e}(t)=\Delta \omega_{\mathrm{n}} t+\alpha_{\mathrm{clk}}\left(t-\tau_{\mathrm{d}}\right)-\alpha_{\mathrm{in}}(t)$. By using (10), the stochastic differential equation describing the total phase error in the loop is obtained by differentiating $\phi_{e}(t)$ and is expressed as

$$
\begin{aligned}
\frac{d \phi_{e}(t)}{d t}=\Delta \omega_{\mathrm{n}}- & \frac{\gamma_{e}\left(t-\tau_{\mathrm{d}}\right)}{\tau_{\mathrm{ch}}} \\
& +\sqrt{c_{\mathrm{clk}}} \Gamma_{\mathrm{clk}}\left(t-\tau_{\mathrm{d}}\right)-\sqrt{c_{\mathrm{in}}} \Gamma_{\mathrm{in}}(t)
\end{aligned}
$$

where $d \alpha_{\mathrm{in}} / d t=\sqrt{c_{\mathrm{in}}} \Gamma_{\mathrm{in}}(t)$, and $\Gamma_{\mathrm{in}}(t)$ is a white noise source associated with the input data signal. Inserting (11) in (5), the output from the loop filter becomes

$$
\begin{aligned}
\frac{d \psi_{e}}{d t}=\mathrm{dc} & +\frac{1}{\tau_{1}} \sum_{i=1}^{\infty} \zeta_{i} \sin \left[i \phi_{e}(t)\right]+\frac{\tau_{2}}{\tau_{1}}\left(\sum_{i=1}^{\infty} \zeta_{i} i \cos \left[i \phi_{e}(t)\right]\right) \\
\times & -\Delta \omega_{\mathrm{n}}+\frac{\gamma_{e}\left(t-\tau_{\mathrm{d}}\right)}{\tau_{\mathrm{ch}}} \\
& \left.-\sqrt{c_{\mathrm{clk}}} \Gamma_{\mathrm{clk}}\left(t-\tau_{\mathrm{d}}\right)+\sqrt{c_{\mathrm{in}}} \Gamma_{\mathrm{in}}(t)\right) .
\end{aligned}
$$

The dynamical behavior of the PLL in the presence of noise is governed by (10)-(12), where dc $=\left(\operatorname{ARGs}_{0}^{\prime} c_{0}^{\prime} / \tau_{1}\right)$, and $\zeta_{i}=$ $2 \mathrm{ARGs}_{i} c_{i \cdot m} \cdot \zeta_{i}$ describes the overall gain in the loop.

\section{LINEARIZATION OF LANGEVIN EQUATIONS}

\section{A. Zero Time Delay}

In this section, it is assumed the time delay is set to zero, i.e., $\tau_{\mathrm{d}}=0$. We want to linearize the Langevin (10)-(12) by 
performing a small signal expansion near a stationary point; $(\bar{x}, \bar{y}, \bar{z}) \equiv\left(\bar{\phi}_{e}, \bar{\gamma}_{e}, \bar{\psi}_{e}\right)=0$. For convenience, we introduce

$$
\begin{aligned}
\phi_{e}(t) & =\bar{x}+\Delta x(t) \\
\gamma_{e}(t) & =\bar{y}+\Delta y(t) \\
\psi_{e}(t) & =\bar{z}+\Delta z(t)
\end{aligned}
$$

where $\Delta x(\cdot), \Delta y(\cdot)$, and $\Delta z(\cdot)$ are small disturbances around the stationary points. Now, the expressions in (13)-(15) are inserted in (10)-(12). After linearization, the following is obtained: ${ }^{1}$

$$
\begin{aligned}
& {\left[\begin{array}{c}
\dot{\Delta} x \\
\dot{\Delta} y \\
\dot{\Delta} z
\end{array}\right]=\left[\begin{array}{ccc}
0 & -\frac{1}{\tau_{\mathrm{ch}}} & 0 \\
0 & -\frac{1}{\tau_{\mathrm{ch}}} & -K_{\mathrm{vco}} \\
\frac{\zeta_{1}}{\tau_{1}} & \frac{\tau_{2} \zeta_{1}}{\tau_{1} \tau_{\mathrm{ch}}} & 0
\end{array}\right]\left[\begin{array}{c}
\Delta x \\
\Delta y \\
\Delta z
\end{array}\right]} \\
& +\left[\begin{array}{ccc}
0 & \sqrt{c_{\mathrm{clk}}} & -\sqrt{c_{\mathrm{in}}} \\
-\sqrt{c_{\mathrm{vco}}} & \sqrt{c_{\mathrm{clk}}} & 0 \\
0 & -\frac{\tau_{2} \zeta_{1}}{\tau_{1}} \sqrt{c_{\mathrm{clk}}} & \frac{\tau_{2} \zeta_{1}}{\tau_{1}} \sqrt{c_{\mathrm{in}}}
\end{array}\right]\left[\begin{array}{c}
\Gamma_{\mathrm{vco}}(t) \\
\Gamma_{\mathrm{clk}}(t) \\
\Gamma_{\mathrm{in}}(t)
\end{array}\right] .
\end{aligned}
$$

To obtain the characteristic equation describing the eigen (natural) solution of the system (without the driving noise terms), we assume solutions of the form $\Delta x=\Delta x_{0} e^{\lambda t}, \Delta y=$ $\Delta y_{0} e^{\lambda t}$, and $\Delta z=\Delta z_{0} e^{\lambda t}$, where $\lambda$ are eigenvalues. The eigenvalues are then governed by the following characteristic equation:

$$
\lambda^{3}+\frac{1}{\tau_{\mathrm{ch}}} \lambda^{2}+\frac{K_{\mathrm{vco}} \zeta_{1} \tau_{2}}{\tau_{1} \tau_{\mathrm{ch}}} \lambda-\frac{\zeta_{1} K_{\mathrm{vco}}}{\tau_{1} \tau_{\mathrm{ch}}}=0 .
$$

\section{B. Nonzero Time Delay}

In the presence of time delay, (10)-(12) include a delay term. The delayed differential equations are harder to deal with, and we would therefore like to transform the delayed differential equations into ordinary differential equations. The method which we use is described in [31] and is valid for relatively small values of the ratio $\tau_{\mathrm{d}} / \tau_{\mathrm{ch}}$, i.e., $\tau_{\mathrm{d}} / \tau_{\mathrm{ch}}<500 \mathrm{~ns}$. Without the driving terms caused by noise, the system of (10)-(12) can be written as

$$
\begin{aligned}
\dot{\mathbf{w}} & =f(\mathbf{w}) \\
\mathbf{w} & =\left[w_{1}(t), w_{2}(t), w_{3}(t), w_{4}(t)\right]^{\mathrm{T}} \\
& \equiv\left[\phi_{e}(t), \gamma_{e}(t), \gamma_{e}\left(t-\tau_{\mathrm{d}}\right), \psi_{e}(t)\right]^{\mathrm{T}} .
\end{aligned}
$$

For each of the variables in $\mathbf{w}=\left[w_{1}(t), w_{2}(t), w_{3}(t)\right.$, $\left.w_{4}(t)\right]^{\mathrm{T}}$, a first-order differential equation needs to be derived in order to get the total response of the loop. The variable $w_{3}(t)$ is a delayed version of $w_{2}(t)$, i.e., $w_{3}(t)=w_{2}\left(t-\tau_{\mathrm{d}}\right)$. Using the first-order Pade $(1,1)$ approximation and following the

\footnotetext{
${ }^{1}$ In practice, $s_{1} c_{m} \gg s_{i} c_{i \cdot m}$ for $i \in[2 ; \infty]$, and only $i=1$ is considered. This is observed by considering the pulse width requirements for the high-speed OTDM data signal [30].
}

procedure described in [31], a differential equation describing $w_{3}(t)$ is obtained:

$$
\frac{d w_{3}}{d t}=\frac{2}{\tau_{\mathrm{d}}}\left[w_{2}(t)-w_{3}(t)\right]-\frac{d w_{2}}{d t} .
$$

Using (19), the differential equations in (10)-(12) are rewritten as (without the noise driving terms)

$$
\begin{aligned}
\frac{d w_{1}}{d t}= & \Delta \omega_{\mathrm{n}}-\frac{w_{3}(t)}{\tau_{\mathrm{ch}}} \\
\frac{d w_{2}}{d t}= & -\frac{w_{2}(t)}{\tau_{\mathrm{ch}}}-K_{\mathrm{vco}} w_{4}(t) \\
\frac{d w_{3}}{d t}= & \frac{2}{\tau_{\mathrm{d}}}\left[w_{2}(t)-w_{3}(t)\right]+\frac{w_{2}(t)}{\tau_{\mathrm{ch}}}+K_{\mathrm{vco}} w_{4}(t) \\
\frac{d w_{4}}{d t}= & \mathrm{dc}+\frac{1}{\tau_{1}} \sum_{i=1}^{\infty} \zeta_{i} \sin \left[i w_{1}(t)\right] \\
& +\frac{\tau_{2}}{\tau_{1}}\left(\sum_{i=1}^{\infty} \zeta_{i} i \cos \left[i w_{1}(t)\right]\right) \cdot\left(-\Delta \omega_{\mathrm{n}}+\frac{w_{3}(t)}{\tau_{\mathrm{ch}}}\right)
\end{aligned}
$$

Next, we want to linearize the Langevin equations in (20)-(23) by performing a small signal expansion near a stationary point; $(\bar{x}, \bar{y}, \bar{q}, \bar{z})=\left(\bar{w}_{1}, \bar{w}_{2}, \bar{w}_{3}, \bar{w}_{4}\right)=0$. Once again, as in (13)-(15), we introduce $w_{1}(t)=\bar{x}+\Delta x(t), w_{2}(t)=$ $\bar{y}+\Delta y(t), w_{3}(t)=\bar{q}+\Delta q(t)$, and $w_{4}(t)=\bar{z}+\Delta z(t)$. After linearization and including the noise terms, the following is obtained:

$$
\begin{aligned}
& {\left[\begin{array}{c}
\dot{\Delta x} \\
\dot{\Delta y} \\
\dot{\Delta q} \\
\dot{\Delta z}
\end{array}\right]=\left[\begin{array}{cccc}
0 & 0 & -\frac{1}{\tau_{\mathrm{ch}}} & 0 \\
0 & -\frac{1}{\tau_{\mathrm{ch}}} & 0 & -K_{\mathrm{vco}} \\
0 & \frac{2}{\tau_{\mathrm{d}}}+\frac{1}{\tau_{\mathrm{ch}}} & -\frac{2}{\tau_{\mathrm{d}}} & K_{\mathrm{vco}} \\
\frac{\zeta_{1}}{\tau_{1}} & 0 & \frac{\tau_{2} \zeta_{1}}{\tau_{1} \tau_{\mathrm{ch}}} & 0
\end{array}\right]\left[\begin{array}{c}
\Delta x \\
\Delta y \\
\Delta q \\
\Delta z
\end{array}\right]} \\
& +\left[\begin{array}{cccc}
0 & 0 & \sqrt{c_{\mathrm{clk}}} & -\sqrt{c_{\mathrm{in}}} \\
-\sqrt{c_{\mathrm{vco}}} & \sqrt{c_{\mathrm{clk}}} & 0 & 0 \\
0 & 0 & 0 & 0 \\
0 & 0 & -\frac{\tau_{2} \zeta_{1}}{\tau_{1}} \sqrt{c_{\mathrm{clk}}} & \frac{\tau_{2} \zeta_{1}}{\tau_{1}} \sqrt{c_{\mathrm{in}}}
\end{array}\right] \\
& \times\left[\begin{array}{c}
\Gamma_{\mathrm{vco}}(t) \\
\Gamma_{\mathrm{clk}}(t) \\
\Gamma_{\mathrm{clk}}\left(t-\tau_{\mathrm{d}}\right) \\
\Gamma_{\mathrm{in}}(t)
\end{array}\right] \text {. }
\end{aligned}
$$

Following the same principle as in Section III-A, the eigenvalues are then governed by the following characteristic equation:

$$
\begin{aligned}
\lambda^{4}+\frac{\left(2 \tau_{1} \tau_{\mathrm{ch}}+\tau_{1} \tau_{\mathrm{d}}\right)}{\left(\tau_{\mathrm{d}} \tau_{1} \tau_{\mathrm{ch}}\right)} \lambda^{3}+\frac{\left(2 \tau_{1}-K_{\mathrm{vco}} \zeta_{1} \tau_{2} \tau_{\mathrm{d}}\right)}{\left(\tau_{\mathrm{d}} \tau_{1} \tau_{\mathrm{ch}}\right)} \lambda^{2} \\
+\frac{\left(2 K_{\mathrm{vco}} \zeta_{1} \tau_{2}-\zeta_{1} K_{\mathrm{vco}} \tau_{\mathrm{d}}\right)}{\left(\tau_{\mathrm{d}} \tau_{1} \tau_{\mathrm{ch}}\right)} \lambda-\frac{2 \zeta_{1} K_{\mathrm{vco}}}{\left(\tau_{\mathrm{d}} \tau_{\mathrm{ch}} \tau_{1}\right)=0}
\end{aligned}
$$

\section{COMPutation OF CORRELATION FunCtions}

In the previous section, we have derived a set of stochastic nonlinear differential (Langevin) equations describing the 
phase error in the loop and furthermore linearized them. In this section, we determine the correlation functions, which will be subsequently used to compute the frequency spectrum of the recovered clock signal and the resulting timing jitter. In short notation, the system of equations in (16) and (24) can be expressed as

$$
\frac{d \mathbf{X}}{d t}=-\mathbf{A X}+\mathbf{D} \Gamma(t)
$$

where $\mathbf{A}$ and $\mathbf{D}$ are constant matrices, and $\Gamma$ denotes a vector of white noise sources with following correlation functions: $\left\langle\Gamma_{i}\left(t_{1}\right) \Gamma_{i}\left(t_{2}\right)\right\rangle=\delta\left(t_{1}-t_{2}\right)$, where $i$ is an integer. We are going to assume vanishing cross correlations:

$$
\begin{aligned}
\left\langle\Gamma_{\text {in }}\left(t_{1}\right) \Gamma_{\mathrm{clk}}\left(t_{2}\right)\right\rangle & =\left\langle\Gamma_{\text {in }}\left(t_{1}\right) \Gamma_{\mathrm{vco}}\left(t_{2}\right)\right\rangle \\
& =\left\langle\Gamma_{\mathrm{vco}}\left(t_{1}\right) \Gamma_{\mathrm{clk}}\left(t_{2}\right)\right\rangle \\
& =0 .
\end{aligned}
$$

Taking into into account that the considered (white) noise sources are stationary, the delayed noise term $\Gamma_{\mathrm{clk}}\left(t-\tau_{\mathrm{d}}\right)$ can be considered as a separate noise source $\Gamma_{\mathrm{d}}(t)$. Equation (26) is easily solved by Fourier transformation technique. Introducing the Fourier transform $X(\omega)$ of $x(t)$

$$
X(\omega)=\int_{-\infty}^{\infty} x(t) e^{-j \omega t} d t \Leftrightarrow x(t)=\frac{1}{2 \pi} \int_{-\infty}^{\infty} X(\omega) e^{j \omega t} d \omega
$$

and using the inverse Fourier transformation, (26) is solved:

$$
\mathbf{X}(\omega)=(\mathbf{A}+j \omega \mathbf{I})^{-1} \mathbf{D} \Gamma(\omega)
$$

where $\mathbf{I}$ is the unity matrix. Since $d \alpha_{\mathrm{in}}(t) / d t=\sqrt{c_{\mathrm{in}}} \Gamma_{\mathrm{in}}(t)$, we have

$$
\alpha_{\mathrm{in}}(\omega)=\frac{\sqrt{c_{\mathrm{in}}} \Gamma_{\mathrm{in}}(\omega)}{j \omega} .
$$

Next, we want to determine the cross correlation functions $\langle\mathbf{x}(\tau) \mathbf{x}(0)\rangle$, where we have assumed that $\mathbf{x}(t)$ is a real widesense-stationary process. This is achieved by first computing the cross-spectral densities $\left\langle\mathbf{X}(\omega)\left(\mathbf{X}^{*}(\omega)\right)^{\mathrm{T}}\right\rangle$ and then transforming to the time domain. The cross-spectral densities are expressed as

$$
\begin{aligned}
\mathbf{S}(\omega) & \equiv\left\langle\mathbf{X}(\omega)\left(\mathbf{X}^{*}(\omega)\right)^{\mathrm{T}}\right\rangle \\
& =\left\langle(\mathbf{A}+j \omega \mathbf{I})^{-1} \mathbf{D} \boldsymbol{\Gamma}(\omega)\left[(\mathbf{A}-j \omega \mathbf{I})^{-1} \mathbf{D} \Gamma^{*}(\omega)\right]^{\mathrm{T}}\right\rangle \\
& =2 \pi(\mathbf{A}+j \omega \mathbf{I})^{-1} \mathbf{D} \mathbf{D}^{\mathrm{T}}\left(\mathbf{A}^{\mathrm{T}}-j \omega \mathbf{I}\right)^{-1} .
\end{aligned}
$$

We want to determine the elements $s_{l m}$ of the matrix $\mathbf{S}(\omega)$, where $l$ and $m$ are integers. Let us define unit vectors $\hat{\mathbf{e}}_{1}^{\mathrm{T}}=$ $\left[\begin{array}{lll}1 & \cdots & 0\end{array}\right], \hat{\mathbf{e}}_{2}^{\mathrm{T}}=\left[\begin{array}{lll}0 & 1 & 0\end{array}\right]$, and $\hat{\mathbf{e}}_{\mathrm{n}}^{\mathrm{T}}=\left[\begin{array}{lll}0 & \cdots & 1\end{array}\right]$. The eigenvectors $\mathbf{V}_{j}$ corresponding to the eigenvalues $\lambda_{j}$ of the matrix $\mathbf{A}^{\mathrm{T}}$ satisfy $\mathbf{A}^{\mathrm{T}} \mathbf{V}_{j}=\lambda_{j} \mathbf{V}$. Using the eigenvectors, we define a new basis in which the unit vectors are expressed as

$$
\begin{aligned}
\hat{\mathbf{e}}_{k} & =b_{k_{1}}\left[\begin{array}{c}
v_{11}^{1} \\
v_{12}^{1} \\
\vdots \\
v_{1 n}^{1}
\end{array}\right]+b_{k_{2}}\left[\begin{array}{c}
v_{11}^{2} \\
v_{12}^{2} \\
\vdots \\
v_{1 n}^{2}
\end{array}\right]+\cdots+b_{k_{\mathrm{n}}}\left[\begin{array}{c}
v_{11}^{n} \\
v_{12}^{n} \\
\vdots \\
v_{1 n}^{n}
\end{array}\right] \\
& =b_{k_{1}} \mathbf{V}_{1}+b_{k_{2}} \mathbf{V}_{2}+\cdots+b_{k_{\mathrm{n}}} \mathbf{V}_{\mathrm{n}}
\end{aligned}
$$

where $k=1, \ldots, n$ and $b_{k_{1}}, \ldots, b_{k_{\mathrm{n}}}$ are constants. The coefficients $s_{l m}$ of the matrix $\mathbf{S}(\omega)$ can now be expressed as

$$
\begin{aligned}
s_{l m}= & \hat{\mathbf{e}}_{l}^{\mathrm{T}} \mathbf{S} \hat{\mathbf{e}}_{m} \\
= & 2 \pi\left(b_{l_{1}} \mathbf{V}_{1}^{\mathrm{T}}+\cdots+b_{l_{\mathrm{n}}} \mathbf{V}_{\mathrm{n}}^{\mathrm{T}}\right)(\mathbf{A}+j \omega \mathbf{I})^{-1} \mathbf{D D}^{\mathrm{T}} \\
& \cdot\left(\mathbf{A}^{\mathrm{T}}-j \omega \mathbf{I}\right)^{-1}\left(b_{m_{1}} \mathbf{V}_{1}+\cdots+b_{m_{\mathrm{n}}} \mathbf{V}_{\mathrm{n}}\right) \\
= & \sum_{i=1}^{n} \sum_{k=1}^{n} 2 \pi b_{l_{i}} \mathbf{V}_{i}^{\mathrm{T}} \\
& \times\left[(\mathbf{A}+j \omega \mathbf{I})^{-1} \mathbf{D D}^{\mathrm{T}} \cdot\left(\mathbf{A}^{\mathrm{T}}-j \omega \mathbf{I}\right)^{-1}\right] \mathbf{V}_{k} b_{m_{k}}
\end{aligned}
$$

where $n$ is the order of the system depicted in (26). We recall that $\left(\mathbf{A}^{\mathrm{T}}\right)^{-1} \mathbf{V}_{j}=\left(1 / \lambda_{j}\right) \mathbf{V}_{j}$ and $\mathbf{V}_{j}^{\mathrm{T}} \mathbf{A}^{-1}=\left(1 / \lambda_{j}\right) \mathbf{V}_{j}^{\mathrm{T}}$. The matrix coefficients $s_{l m}$ are therefore expressed as

$$
\begin{aligned}
s_{l m}= & \sum_{i=1}^{n} \sum_{k=1}^{n} \frac{2 \pi b_{l_{i}} \mathbf{V}_{i}^{\mathrm{T}} \mathbf{D} \mathbf{D}^{\mathrm{T}} \mathbf{V}_{k} b_{m_{k}}}{\left(\lambda_{i}+j \omega\right)\left(\lambda_{k}-j \omega\right)} \\
= & \sum_{i=1}^{n} \sum_{k=1}^{n} \frac{2 \pi b_{l_{i}} \mathbf{V}_{i}^{\mathrm{T}} \mathbf{D} \mathbf{D}^{\mathrm{T}} \mathbf{V}_{k} b_{m_{k}}}{\left(\lambda_{i}+\lambda_{k}\right)} \\
& \times\left(\frac{1}{\lambda_{k}-j \omega}+\frac{1}{\lambda_{i}+j \omega}\right) .
\end{aligned}
$$

Taking the inverse Fourier transformation of (34), the cross correlation functions are found as follows:

$$
\begin{aligned}
s_{l m}(\tau)= & \left\langle x_{l}(\tau) x_{m}(0)\right\rangle \\
= & \sum_{i=1}^{n} \sum_{k=1}^{n} \frac{b_{l_{i}} \mathbf{V}_{i}^{\mathrm{T}} \mathbf{D D}^{\mathrm{T}} \mathbf{V}_{k v} b_{m_{k}}}{\left(\lambda_{i}+\lambda_{k}\right)} \\
& \times\left(e^{\lambda_{k} \tau} \mu(-\tau)+e^{-\lambda_{i} \tau} \mu(\tau)\right) .
\end{aligned}
$$

Since the matrix $\mathbf{D} \mathbf{D}^{\mathrm{T}}$ is symmetric, we have $\mathbf{V}_{i}^{\mathrm{T}} \mathbf{D} \mathbf{D}^{\mathrm{T}} \mathbf{V}_{k}=$ $\left(\mathbf{V}_{i}^{\mathrm{T}} \mathbf{D} \mathbf{D}^{\mathrm{T}} \mathbf{V}_{k}\right)^{\mathrm{T}}=\mathbf{V}_{k}^{\mathrm{T}} \mathbf{D} \mathbf{D}^{\mathrm{T}} \mathbf{V}_{i}$. When $l=m$, (35) can then be expressed as

$$
\begin{aligned}
s_{m m}(\tau)= & \sum_{i=1}^{n}\left(\frac{b_{m_{i}} \mathbf{V}_{i}^{\mathrm{T}} \mathbf{D} \mathbf{D}^{\mathrm{T}} \mathbf{V}_{i} b_{m_{i}}}{2 \lambda_{i}}\right. \\
& \left.\quad+\sum_{j \neq i, j=1}^{n} \frac{b_{m_{i}} \mathbf{V}_{i}^{\mathrm{T}} \mathbf{D D}^{\mathrm{T}} \mathbf{V}_{j} b_{m_{j}}}{\lambda_{i}+\lambda_{j}}\right) \cdot e^{-\lambda_{i}|\tau|} \\
= & \sum_{i=1}^{n} \nu_{i}^{m m} e^{-\lambda_{i}|\tau|}
\end{aligned}
$$


where $\mu(\cdot)$ is the unit step function. Furthermore, when $l \neq m$, $s_{l m}(\tau)+s_{m l}(\tau)$ can be expressed as

$$
\begin{aligned}
s_{l m}(\tau)+s_{m l}(\tau)= & 2 \sum_{i=1}^{n}\left(\frac{b_{l_{i}} \mathbf{V}_{i}^{\mathrm{T}} \mathbf{D} \mathbf{D}^{\mathrm{T}} \mathbf{V}_{i} b_{m_{i}}}{2 \lambda_{i}}\right. \\
& \left.+\sum_{j \neq i, j=1}^{n} \frac{b_{l_{i}} \mathbf{V}_{i}^{\mathrm{T}} \mathbf{D} \mathbf{D}^{\mathrm{T}} \mathbf{V}_{j} b_{m_{j}}}{\lambda_{i}+\lambda_{j}}\right) \\
& \cdot e^{-\lambda_{i}|\tau|} \\
= & 2 \sum_{i=1}^{n} \nu_{i}^{l m} e^{-\lambda_{i}|\tau|} .
\end{aligned}
$$

Next, we determine the correlation function $\left\langle\alpha_{\text {in }}(\tau) \mathbf{x}(0)\right\rangle$. We use the same procedure as before and first determine the cross-spectral densities

$$
\begin{aligned}
\left\langle\alpha_{\mathrm{in}}(\omega) \mathbf{X}^{*}(\omega)\right\rangle & =\left\langle\frac{\sqrt{c_{\mathrm{in}}} \Gamma_{\mathrm{in}}(\omega)}{j \omega}(\mathbf{A}-j \omega \mathbf{I})^{-1} \mathbf{D} \Gamma^{*}(\omega)\right\rangle \\
& =\frac{2 \pi \sqrt{c_{i n}}}{j \omega}(\mathbf{A}-j \omega \mathbf{I})^{-1} \mathbf{D} \hat{\mathbf{e}}_{\mathrm{n}} .
\end{aligned}
$$

The correlation functions $\left\langle\alpha_{\text {in }} x_{l}^{*}(\omega)\right\rangle$, where $x_{l}^{*}(\omega)$ are the elements of $\mathbf{X}^{*}(\omega)$, are expressed as

$$
\begin{aligned}
\left\langle\alpha_{\mathrm{in}}(\omega) x_{l}^{*}(\omega)\right\rangle & =\frac{2 \pi \sqrt{c_{\mathrm{in}}}}{j \omega} \hat{\mathbf{e}}_{l}^{\mathrm{T}}(\mathbf{A}-j \omega \mathbf{I})^{-1} \mathbf{D} \hat{\mathbf{e}}_{\mathrm{n}} \\
& =\sum_{i=1}^{n} \frac{2 \pi \sqrt{c_{\mathrm{in}}} b_{l_{i}} \mathbf{V}_{i}^{\mathrm{T}}(\mathbf{A}-j \omega \mathbf{I})^{-1} \mathbf{D} \hat{\mathbf{e}}_{\mathrm{n}}}{j \omega} \\
& =\sum_{i=1}^{n} \frac{2 \pi \sqrt{c_{\mathrm{in}}} b_{l_{i}} \mathbf{V}_{i}^{\mathrm{T}} \mathbf{D} \hat{\mathbf{e}}_{\mathrm{n}}}{\omega^{2}+j \omega \lambda_{i}}
\end{aligned}
$$

Similarly, it can be shown that $\left\langle x_{l}(\omega) \alpha_{\mathrm{in}}^{*}(\omega)\right\rangle$ is given by

$$
\left\langle x_{l}(\omega) \alpha_{\mathrm{in}}^{*}(\omega)\right\rangle=\sum_{i=1}^{n} \frac{2 \pi \sqrt{c_{\mathrm{in}}} b_{l_{i}} \mathbf{V}_{i}^{\mathrm{T}} \mathbf{D} \hat{\mathbf{e}}_{\mathrm{n}}}{\omega^{2}-j \omega \lambda_{i}} .
$$

As will be shown in Section $\mathrm{V}$, the correlation function $\left\langle\alpha_{\mathrm{in}} x_{l}^{*}(\omega)\right\rangle$ always contains the pair $\left\langle x_{l}(\omega) \alpha_{\mathrm{in}}^{*}(\omega)\right\rangle$ when computing the frequency spectrum of the clock signal. It is therefore convenient to determine the Fourier transformation of $\left\langle\alpha_{\mathrm{in}}(\omega) x_{l}^{*}(\omega)\right\rangle+\left\langle x_{l}(\omega) \alpha_{\mathrm{in}}^{*}(\omega)\right\rangle$. This is shown to be

$$
\begin{aligned}
\left\langle\alpha_{\mathrm{in}}(\tau) x_{l}(0)\right\rangle+\left\langle x_{l}(\tau) \alpha_{i n}(0)\right\rangle & =\sum_{i=1}^{n} \frac{\sqrt{c_{\mathrm{in}}} b_{l_{i}} \mathbf{V}_{i}^{\mathrm{T}} \mathbf{D} \hat{\mathbf{e}}_{\mathrm{n}}}{\lambda_{i}} e^{-\lambda_{i}|\tau|} \\
& =\sum_{i=1}^{n} \mu_{i}^{l} e^{-\lambda_{i}|\tau|}
\end{aligned}
$$

The constants $b_{l_{i}}$, where $l$ and $i$ are integers, appearing in (36) and (41) are the elements of a vector $\mathbf{B}_{l}$ and are determined by solving the following matrix equation:

$$
\begin{aligned}
\mathbf{B}_{l} & =\mathbf{V}^{-1} \hat{\mathbf{e}}_{l} \\
\mathbf{V} & =\left[\begin{array}{cccc}
v_{11} & v_{21} & \cdots & v_{n 1} \\
v_{12} & v_{22} & \cdots & v_{n 2} \\
\vdots & \vdots & \cdots & \vdots \\
v_{1 n} & v_{2 n} & \cdots & v_{n n}
\end{array}\right] \\
& \equiv\left[\begin{array}{cccc}
v_{11}^{1} & v_{11}^{2} & \cdots & v_{11}^{n} \\
v_{12}^{1} & v_{12}^{2} & \cdots & v_{12}^{n} \\
\vdots & \vdots & \cdots & \vdots \\
v_{1 n}^{1} & v_{1 n}^{2} & \cdots & v_{1 n}^{n}
\end{array}\right]
\end{aligned}
$$

where $\hat{\mathbf{e}}_{l}$ are unit vectors.

\section{Computation of the Autocorrelation Functions of THE EXTRACTED Clock Signals}

The intensity of a locally generated optical clock signal in the presence of phase noise (2) is expressed as

$$
\begin{aligned}
P_{\text {clk }}(t) & =\sum_{q=-\infty}^{\infty} c_{q} e^{j q \omega_{0}^{\prime} t} e^{j q \omega_{0}^{\prime} \alpha_{\text {clk }}(t)} \\
& =\sum_{q=-\infty}^{\infty} c_{q} e^{j q \omega_{0}^{\prime}\left(t+\alpha_{\text {clk }}(t)\right)}
\end{aligned}
$$

where $\omega_{0}^{\prime}=2 \pi\left(f_{0}^{\prime} / m\right)$. The autocorrelation function $\left\langle P_{\text {clk }}(t) P_{\text {clk }}^{*}(t+\tau)\right\rangle$ of the optical clock signal is expressed as

$$
\begin{aligned}
& \left\langle P_{\mathrm{clk}}(t) P_{\mathrm{clk}}^{*}(t+\tau)\right\rangle \\
& \quad=\sum_{q=-\infty}^{q=\infty} \sum_{k=-\infty}^{\infty} c_{q} c_{k}^{*} e^{j(q-k) \omega_{0}^{\prime} t} e^{-j k \omega_{0}^{\prime} \tau}\left\langle e^{j \omega_{0}^{\prime} \varphi_{q k}(t, \tau)}\right\rangle
\end{aligned}
$$

where $\quad \varphi_{q k}(t, \tau)=q \alpha_{\mathrm{clk}}(t)-k \alpha_{\mathrm{clk}}(t+\tau) . \quad$ The term $\left\langle e^{j \omega_{0}^{\prime} \varphi_{q, k}(t, \tau)}\right\rangle$ corresponds to the characteristic function of the stochastic process $q \alpha_{\text {clk }}(t)-k \alpha_{\text {clk }}(t+\tau)$. It has been shown that the characteristic function of the zero mean Gaussian distributed random variable $\Gamma, N\left(0, \sigma_{\Gamma}^{2}\right)$ equals $\left\langle e^{j \omega \Gamma}\right\rangle=e^{-(1 / 2) \omega^{2} \sigma_{\Gamma}^{2}}$ [32]. As stated earlier, the phase noise of the optical clock signal is asymptotically a zero mean wide-sense stationary Gaussian process, and therefore, the following is valid: $\left\langle e^{j \omega_{0}^{\prime} \varphi_{q, k}(t, \tau)}\right\rangle=e^{-(1 / 2) \omega_{0}^{\prime 2} \sigma_{\varphi}^{2}(t, \tau)}$. The variance $\sigma_{\varphi}^{2}(t, \tau)$ is expressed as

$$
\begin{aligned}
\sigma_{\varphi}^{2}(t, \tau)= & \left\langle\left[q \alpha_{\mathrm{clk}}(t)-k \alpha_{\mathrm{clk}}(t+\tau)\right]^{2}\right\rangle \\
& -\left\langle\left[q \alpha_{\mathrm{clk}}(t)-k \alpha_{\mathrm{clk}}(t+\tau)\right]\right\rangle^{2} \\
= & q^{2}\left\langle\alpha_{\mathrm{clk}}^{2}(t)\right\rangle-2 q k\left\langle\alpha_{\mathrm{clk}}(t) \alpha_{\mathrm{clk}}(t+\tau)\right\rangle \\
& +k^{2}\left\langle\alpha_{\mathrm{clk}}^{2}(t+\tau)\right\rangle .
\end{aligned}
$$

Taking the stationarity of the phase noise into consideration, the variance $\sigma_{\varphi}^{2}(t, \tau)$ is given by

$$
\begin{aligned}
\sigma_{\varphi}^{2}(t, \tau)=\left(q^{2}+k^{2}\right)\left\langle\alpha_{\mathrm{clk}}(\tau=\right. & \left.0) \alpha_{\mathrm{clk}}(0)\right\rangle \\
& -2 q k\left\langle\alpha_{\mathrm{clk}}(\tau) \alpha_{\mathrm{clk}}(0)\right\rangle .
\end{aligned}
$$


In order to determine the autocorrelation function of the optical clock signal, the autocorrelation function of the phase noise $\left\langle\alpha_{\mathrm{clk}}(\tau) \alpha_{\mathrm{clk}}(0)\right\rangle$ needs to be calculated. By using the Wiener-Khintchine theorem [33], the autocorrelation function $\left\langle\alpha_{\text {clk }}(\tau) \alpha_{\text {clk }}(0)\right\rangle$ can be expressed in terms of the spectral density

$$
\left\langle\alpha_{\mathrm{clk}}(\tau) \alpha_{\mathrm{clk}}(0)\right\rangle=\frac{1}{2 \pi} \int_{-\infty}^{+\infty} \frac{\left\langle\alpha_{\mathrm{clk}}(\omega) \alpha_{\mathrm{clk}}^{*}(\omega)\right\rangle}{2 \pi} e^{j \omega \tau} d \omega
$$

Using (4), we have that $\alpha_{\mathrm{clk}}(\omega)=\phi_{e}(\omega)+\alpha_{\text {in }}(\omega)$, and it can therefore be shown that the spectral density of the optical clock signal phase noise $\left\langle\alpha_{\mathrm{clk}}(\omega) \alpha_{\mathrm{clk}}^{*}(\omega)\right\rangle$ becomes

$$
\begin{aligned}
\left\langle\alpha_{\mathrm{clk}}(\omega) \alpha_{\mathrm{clk}}^{*}(\omega)\right\rangle= & \left\langle\alpha_{\mathrm{in}}(\omega) \alpha_{\mathrm{in}}^{*}(\omega)\right\rangle+\left\langle\alpha_{\mathrm{in}}(\omega) \phi_{e}^{*}(\omega)\right\rangle \\
& +\left\langle\phi_{e}(\omega) \alpha_{\mathrm{in}}^{*}(\omega)\right\rangle+\left\langle\phi_{e}(\omega) \phi_{e}^{*}(\omega)\right\rangle .
\end{aligned}
$$

In order to compute the autocorrelation function of the optical clock signal phase noise $\left\langle\alpha_{\mathrm{clk}}(\tau) \alpha_{\mathrm{clk}}(0)\right\rangle$, (48) is inserted into (47), and we furthermore use (30), (36), and (41). After inverse Fourier transformation, one gets

$$
\begin{aligned}
\left\langle\alpha_{\mathrm{clk}}(\tau) \alpha_{\mathrm{clk}}(0)\right\rangle= & \left\langle\alpha_{\mathrm{in}}(\tau) \alpha_{\mathrm{in}}(0)\right\rangle+\left\langle\alpha_{\mathrm{in}}(\tau) \phi_{e}(0)\right\rangle \\
& \left.+\left\langle\phi_{e}(\tau) \alpha_{\mathrm{in}}(0)\right\rangle+\left\langle\phi_{e}(\tau) \phi_{e}(0)\right\rangle\right) \\
= & -\frac{1}{2} c_{\mathrm{in}}|\tau|+\sum_{i=1}^{n}\left(\mu_{i}^{1}+\nu_{i}^{11}\right) e^{-\lambda_{i}|\tau|} .
\end{aligned}
$$

The variance of the phase noise $\sigma_{\varphi}^{2}(t, \tau)$ is obtained by inserting (49) into (46):

$$
\begin{aligned}
\sigma_{\varphi}^{2}(t, \tau)=q k c_{\mathrm{in}}|\tau|+\left(q^{2}+k^{2}\right) & \sum_{i=1}^{n}\left(\mu_{i}^{1}+\nu_{i}^{11}\right) \\
& -2 q k \sum_{i=1}^{n}\left(\mu_{i}^{1}+\nu_{i}^{11}\right) e^{-\lambda_{i}|\tau|} .
\end{aligned}
$$

Inserting the expression for the variance (50) into (44) and observing that only terms corresponding to $k=q$ are nonzero for $t \rightarrow \infty$, the autocorrelation function of the optical clock signal $\left\langle P_{\text {clk }}(t) P_{\text {clk }}^{*}(t+\tau)\right\rangle$ is expressed as

$$
\begin{aligned}
& \left\langle P_{\mathrm{clk}}(t) P_{\mathrm{clk}}^{*}(t+\tau)\right\rangle=\sum_{q=-\infty}^{\infty}\left|c_{q}\right|^{2} e^{-j q \omega_{0}^{\prime} \tau} \\
& \cdot \exp \left(-\frac{1}{2} q^{2} \omega_{0}^{\prime 2}\left[c_{\mathrm{in}}|\tau|+2 \sum_{i=1}^{n}\left(\mu_{i}^{1}+\nu_{i}^{11}\right)\left(1-e^{-\lambda_{i}|\tau|}\right)\right]\right) .
\end{aligned}
$$

Next, we determine the autocorrelation function of the VCO signal $\left\langle v(t) v^{*}(t+\tau)\right\rangle$, enabling a comparison between the extracted optical and electrical clock signal. Following the procedure above, and expressing $\alpha_{\mathrm{vco}}(t)=\alpha_{\text {in }}(t)+\phi_{e}(t)-\gamma_{e}(t)$, the autocorrelation function becomes (52), shown at the bottom of the page.

The frequency spectrum of the extracted optical and electrical clock signal can now be obtained by taking the Fourier transform of (51) and (52), respectively.

\section{PDF OF CLOCK SignAL}

Using the autocorrelation functions determined in the previous section, we determine the pdf of the extracted clock signal. In general, given a stochastic process $x(t)$, we form another stochastic process by the following definition:

$$
y(t)=T[x(t)] .
$$

The process $y(t)$ is completely specified in terms of the operator $T$ and $x(t)$. The pdf of $y(t),\left(p_{y}(y ; t)\right)$ can be determined in terms of the density of $x(t),\left(p_{x}(x ; t)\right)$ [32]

$$
p_{y}(y ; t)=\sum_{\mathrm{n}} \frac{p_{x}\left(x_{\mathrm{n}} ; t\right)}{\left|y^{\prime}\left(x_{\mathrm{n}} ; t\right)\right|}
$$

where $x_{\mathrm{n}}$ are the roots of the equation $y(t)-T\left[x_{\mathrm{n}}\right]=0$. Let us now consider the stochastic process $\alpha_{\mathrm{clk}}(t)$ and the corresponding transformation:

$$
y(t)=\sum_{k=-\infty}^{\infty} A_{0} e^{-\left[\frac{t_{k}+\alpha_{\text {clk }}(t)}{T_{0}}\right]^{2}}
$$

where $A_{0}$ is a constant, $t_{k}=t+k T_{p}$, and $T_{p}=\left(1 / f_{0}^{\prime}\right)$. Equation (55) represents a Gaussian pulse train (optical clock signal) corrupted with phase noise. By using (54), we will determine the pdf of the optical clock signal, i.e., $P_{y}(y ; t)$. Since $\alpha_{\text {clk }}(t)$ is (asymptotically) a zero mean wide-sense stationary Gaussian process, the pdf $P_{\alpha_{\mathrm{clk}}}\left(\alpha_{\mathrm{clk}} ; t\right)$ is expressed as

$$
P_{\alpha_{\mathrm{clk}}}\left(\alpha_{\mathrm{clk}} ; t\right)=\frac{1}{\sqrt{2 \pi} \sigma_{\alpha_{\mathrm{clk}}}} e^{-\frac{\alpha_{\mathrm{clk}}^{2}}{2 \sigma_{\alpha_{\mathrm{clk}}}}}
$$

where the variance $\sigma_{\alpha_{\mathrm{clk}}}^{2}$ is determined from the autocorrelation function (49) by setting $\tau$ to zero, i.e., $\sigma_{\alpha_{\text {clk }}}^{2}=\sum_{i=1}^{n}\left(\mu_{i}^{1}+\right.$ $\left.\nu_{i}^{11}\right)$. The roots $x_{\mathrm{n}} \equiv \alpha_{\mathrm{clk}_{\mathrm{n}}}$ are obtained by solving the following equation:

$$
y(t)-\sum_{k=-\infty}^{\infty} A_{0} e^{-\left[\frac{t_{k}+\alpha_{\mathrm{clk}}}{T_{0}}\right]^{2}}=0 .
$$

$$
\left\langle v(t) v^{*}(t+\tau)\right\rangle=\sum_{p=-1}^{1} \frac{V_{0}^{2}}{4} e^{-j p \omega_{0}^{\prime} \tau} \exp \left(-\frac{1}{2} p^{2} \omega_{0}^{\prime 2}\left[c_{\text {in }}|\tau|+2 \sum_{i=1}^{n}\left(\mu_{i}^{1}-\mu_{i}^{2}+\nu_{i}^{11}+\nu_{i}^{22}-2 \nu_{i}^{12}\right)\left(1-e^{-\lambda_{i}|\tau|}\right)\right]\right)
$$


We want to determine the solution for $0<y(t) \leq A_{0}$. Equation (57) is easily solved numerically. For an isolated Gaussian pulse, by setting $k=0$ in (55), the roots $\alpha_{\mathrm{clk}_{1,2}}$ are easily determined:

$$
y(t)=A_{0} e^{-\left[\frac{t+\alpha_{\mathrm{clk}_{\mathrm{n}}}}{T_{0}}\right]^{2}} \Rightarrow \alpha_{\mathrm{clk}_{1,2}}=-t \pm T_{0} \sqrt{\ln \left(\frac{A_{0}}{y(t)}\right)} .
$$

If we assume that the $T_{\mathrm{FWHM}}$ of the optical clock pulse signal satisfies ${ }^{2} T_{\mathrm{FWHM}} \ll T_{p}$, then the roots $\alpha_{\mathrm{clk}_{\mathrm{n}}}$ of (57) can be expressed as

$$
\alpha_{\mathrm{clk}_{\mathrm{n}}}=-\left(t+k T_{p}\right) \pm T_{0} \sqrt{\ln \left(\frac{A_{0}}{y(t)}\right)} .
$$

Now, the pdf of an optical clock signal can be determined by using (54) and (59):

$$
\begin{aligned}
P_{y}(y ; t) & =\sum_{k=-\infty}^{\infty} \frac{T_{0}}{2 \sqrt{2 \pi} \sigma_{\alpha_{\mathrm{clk}}} y \sqrt{\ln \left(A_{0} / y\right)}} \\
\times & \left(e^{-\frac{\left(-t_{k}+T_{0} \sqrt{\left.\ln \left(A_{0} / y\right)\right)^{2}}\right.}{2 \sigma_{\alpha_{\mathrm{clk}}}^{2}}}+e^{-\frac{\left(-t_{k}-T_{0} \sqrt{\left.\ln \left(A_{0} / y\right)\right)^{2}}\right.}{2 \sigma_{\alpha_{\mathrm{clk}}}^{2}}}\right) .
\end{aligned}
$$

The pdf calculated in (60) shows that even though the phase noise $\alpha_{\mathrm{clk}}(t)$ has a Gaussian distribution, after the transformation the pdf of the optical clock signal is not Gaussian. Next, the mean of the optical clock signal is computed. In general, the mean of the stochastic process $y(t)$ specified in (53) is given by the following integral [32]:

$$
\langle y(t)\rangle=\int_{-\infty}^{\infty} T\left[\alpha_{c l k}(t)\right] p_{\alpha_{\mathrm{clk}}}\left(\alpha_{\mathrm{clk}} ; t\right) d \alpha_{\mathrm{clk}} .
$$

In order to compute the mean value of the optical clock signal, we therefore need to solve

$$
\langle y(t)\rangle=\frac{A_{0}}{\sqrt{2 \pi} \sigma_{\alpha_{\mathrm{clk}}}} \sum_{k=-\infty}^{k=\infty} \int_{-\infty}^{\infty} e^{-\left[\frac{t_{k}+\alpha_{\mathrm{clk}}}{T_{0}}\right]^{2}} e^{-\frac{\alpha_{\mathrm{clk}}^{2}}{2 \sigma_{\alpha_{\mathrm{clk}}}^{2}}} d \alpha_{\mathrm{clk}} .
$$

The integral in (62) is easily solved, and the mean of the optical clock signal is thereby given as

$$
\langle y(t)\rangle=\sum_{k=-\infty}^{k=\infty} \frac{A_{0} T_{0}}{\sqrt{\left(T_{0}^{2}+2 \sigma_{\alpha_{\mathrm{clk}}}^{2}\right)}} e^{-\frac{t_{k}^{2}}{\left(T_{0}^{2}+2 \sigma_{\alpha_{\mathrm{clk}}}^{2}\right)}} .
$$

Having computed the mean value of the extracted optical clock signal the corresponding variance is computed by setting $\tau$ to zero in (51) and using (63). By using the same procedure, one can obtain the pdf and mean of the extracted VCO signal.

\footnotetext{
${ }^{2}$ In practice, this condition will be satisfied. Typically, the repetition frequency of the optical clock signal will be 40 or $10 \mathrm{GHz}$. The corresponding period time $T_{p}$ is therefore 25 or $100 \mathrm{ps}$, and the typical values of the $T_{\mathrm{FWHM}}$ are in the range from $1-5 \mathrm{ps}$.
}

TABLE I

Parameters of the OPLL-Based CR That Are Held Constant THROUGHOUT THE SimULATIONS

\begin{tabular}{|l|r|}
\hline Input sig. freq. & $160 \mathrm{GHz}$ \\
\hline Free-running laser freq. & $10 \mathrm{GHz}$ \\
\hline Free-running VCO freq. & $10 \mathrm{GHz}$ \\
\hline Input sig. power & $15 \mathrm{dBm}$ \\
\hline Laser sig. power & $15 \mathrm{dBm}$ \\
\hline VCO sig. amplitude & $1 \mathrm{~V}$ \\
\hline Photodiode resp. & $0.95 \mathrm{~A} / \mathrm{W}$ \\
\hline VCO gain & $10^{5} \mathrm{~Hz} / \mathrm{V}$ \\
\hline
\end{tabular}

\section{RESULTS}

In Table I, we have summarized the key system parameters that are held fixed throughout the simulations.

\section{A. Timing Jitter as a Function of Loop Gain}

In this section, it is assumed that the laser characteristic knee frequency $f_{\text {ch }}$ is large enough such that phase noise of the VCO is completely transferred to the laser, i.e., $\alpha_{\mathrm{clk}}(t)=\alpha_{\mathrm{vco}}(t)$ and thereby, $\gamma_{e}(t)=0$. We therefore only need to consider the phase noise associated with the input signal $\alpha_{\text {in }}(t)$ and the $\operatorname{VCO} \alpha_{\mathrm{vco}}(t)$.

In practice, we are usually interested in the spectrum of the recovered clock signal $S(f)$ around the first harmonic, i.e., $f_{0}^{\prime} / m$ or $f_{0}^{\prime \prime}$. The single-sideband to carrier ratio (SSCR) (in $\mathrm{dBc} / \mathrm{Hz}$ ) is very widely used in practice to characterize the noise performance of the extracted clocks signal [2]. The SSCR is defined as [26]

$$
\mathrm{SSCR} \equiv 10 \log _{10}\left(\frac{S\left(f_{0}^{\prime} / m+f\right)}{|X|}\right)
$$

where $f$ is the offset frequency from the first harmonic, and $|X|=\left|c_{1}\right|^{2}$ or $V_{0}^{2} / 2$. The SSCR gives the amount of phase noise around the first harmonic. In the absence of phase noise, the extracted clock signal frequency spectrum $S(f)$ would only contain discrete frequency component at $k f_{0}^{\prime} / m$ or $k f_{0}^{\prime \prime}$, where $k$ is an integer.

We want to investigate how the SSCR of the extracted clock signal, at the VCO output, is affected by the input data and VCO signal phase noise, loop parameters, and time delay. Furthermore, timing jitter of the clock signal is computed by integrating the spectrum of the recovered clock signal: see (71). Initially, we assume that the VCO signal contains more phase noise than the input data signal, i.e., $c_{\mathrm{vco}}>c_{\mathrm{in}}$. Time delay is set to zero.

In Fig. 2(a), the SSCR of the extracted $10-\mathrm{GHz}$ clock signal, around the first harmonic (i.e., $10 \mathrm{GHz}$ ), is computed when the overall loop gain $\xi=\zeta_{1} K_{\mathrm{vco}}$ is varied from $10^{4}$ to $10^{8} \mathrm{rad} \cdot \mathrm{A} / \mathrm{s} \cdot \mathrm{V}$. These values of the loop gain are obtainable in practical realizations of the optical PLL using standard components. For low frequencies, the clock SSCR follows the input data signal SSCR, and for higher frequencies, it follows the SSCR of the VCO. The offset frequency, at which the SSCR of the clock signal makes a transition to the SSCR of the 


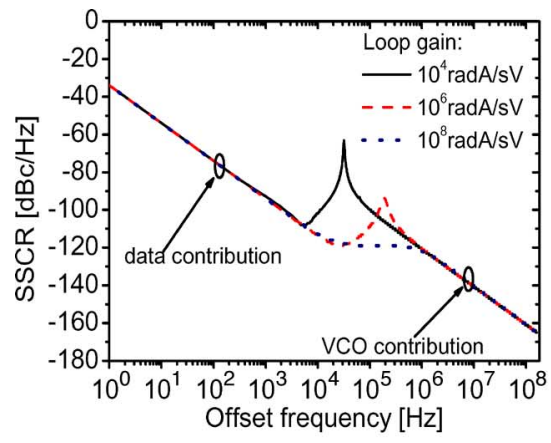

(a)

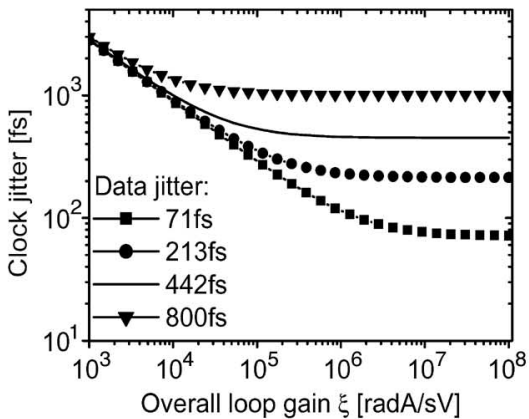

(b)

Fig. 2. (a) SSCR of the extracted clock signal for selected values of the overall loop gain. (b) Timing jitter of the extracted clock signal as a function of the overall loop gain for selected values of input data signal timing jitter. The VCO jitter is $2.25 \mathrm{ps}$, and PI filter bandwidth is $20 \mathrm{MHz}$.

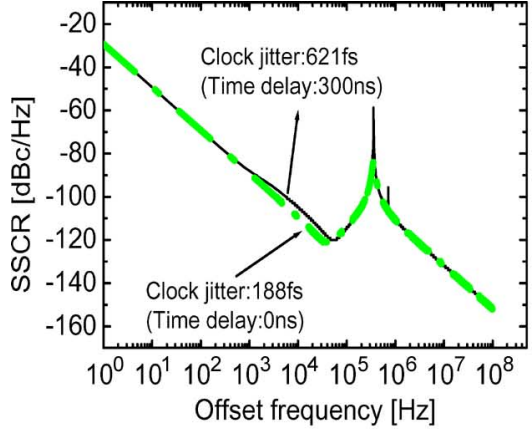

(a)

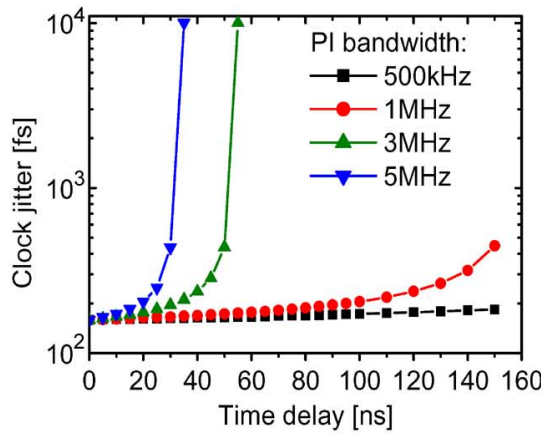

(b)

Fig. 3. (a) SSCR of the extracted clock signal for zero and 300-ns time delay. (b) Clock jitter as a function of time delay for selected PI filter bandwidths.

VCO, corresponds to the bandwidth of the OPLL. This implies that the low-frequency timing jitter is directly transferred from the input OTDM data signal to the clock signal, while the high-frequency timing jitter of the clock signal originates from the VCO. In general, we are interested in reducing the highfrequency jitter from the clock signal since it may result in a penalty when using the clock signal for optical gating. As the overall loop gain $\xi$ is increased from $10^{4}$ to $10^{8} \mathrm{rad} \cdot \mathrm{A} / \mathrm{s} \cdot \mathrm{V}$, the SSCR of the clock signal becomes less and less influenced by the VCO signal. The sideband is pushed down and away, as experimentally verified in [34]. For sufficiently large values of the overall loop gain, the SSCR of the clock signal will approach the SSCR of the input data signal. This is also shown in Fig. 2(b), where timing jitter of the extracted clock signal (jitter integration range: $1 \mathrm{~Hz}-5 \mathrm{GHz}$ ) is plotted as a function of the overall loop gain, for input OTDM data signal jitter in the range from 71 to $800 \mathrm{fs}$ (jitter integration range: $1 \mathrm{~Hz}-80 \mathrm{GHz}$ ). Equation (72) is used to compute the timing jitter of the input signal. Increasing the loop gain thus reduces the timing jitter of the extracted clock signal when $c_{\mathrm{vco}}>c_{\mathrm{in}}$ : see Fig. 2(b). As the overall loop gain is increased sufficiently, the clock timing jitter approaches its minimum value. It is worth remarking that this minimum value corresponds to the input OTDM data signal jitter. However, when the input data signal jitter is relatively large, i.e., $800 \mathrm{fs}$, the minimum obtainable jitter of the clock signal is approximately $1 \mathrm{ps}$. In practice, the PLL overshoots if the loop gain is increased unconditionally. Since the PLL is modeled in the small signal regime (Ornstein-Uhlenbeck process), the overshooting can therefore not be observed in the analytical results [38].

\section{B. Timing Jitter in the Presence of Time Delay}

Until now, we assumed that the time delay in the loop was zero. The influence of a time delay on the timing jitter of the extracted clock signal is investigated by looking at the SSCR of the extracted clock signal for zero and 300-ns time delay ( $\sim 60 \mathrm{~m}$ ); see Fig. 3(a). Notice that the SSCR of the extracted clock signal increases, around the resonant peak, when the time delay is $300 \mathrm{~ns}$, compared to the zero time delay case. This is similar to the experimental observations reported in [37] and [38]. An increase in time delay from 0 to $300 \mathrm{~ns}$ results in an increased timing jitter from 188 to $621 \mathrm{fs}$. In the presence of time delay, the behavior of the loop is very much dependent on the PI filter bandwidth [22]. We therefore need to investigate the timing jitter dependence of the time delay as the PI filter bandwidth is varied. This is shown in Fig. 3(b). The clock jitter increases as the time delay increases. However, this is most pronounced for the PI filter bandwidth $f_{\mathrm{bw}}$ of 3 and $5 \mathrm{MHz}$. The loop's dynamical behavior becomes more unstable, resulting in increased timing jitter as the time delay approaches its critical value $\left(\tau_{\mathrm{d}}=1 / 2 f_{\mathrm{bw}}\right)$ [22]. The impact of time delay on timing jitter is negligible, as long as we are far away from the critical value.

Next, we investigate how the timing jitter of the extracted clock is affected by a time delay as we vary the input data and 


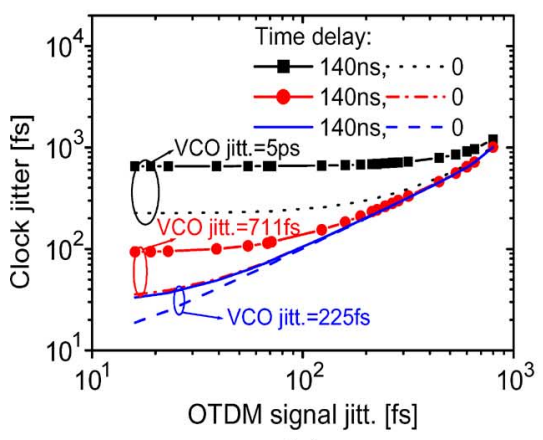

(a)

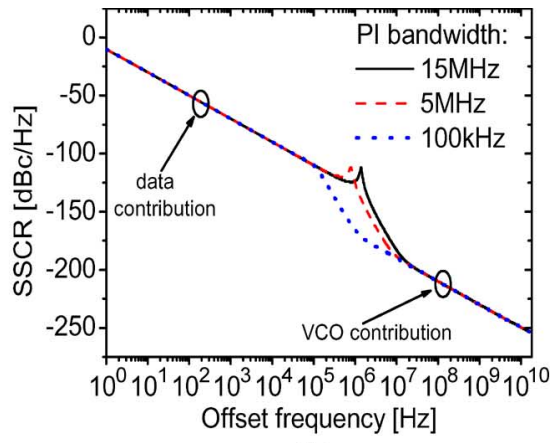

(b)

Fig. 4. (a) Jitter transfer function for zero and 140-ns time delay. PI filter bandwidth: $1 \mathrm{MHz}$. (b) SSCR of the clock signal for selected values of the PI filter bandwidths. Input signal jitter: $990 \mathrm{fs}$. VCO signal jitter: $70 \mathrm{fs}$.

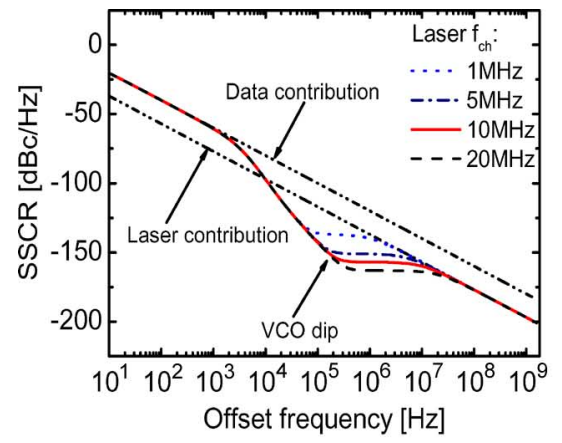

(a)

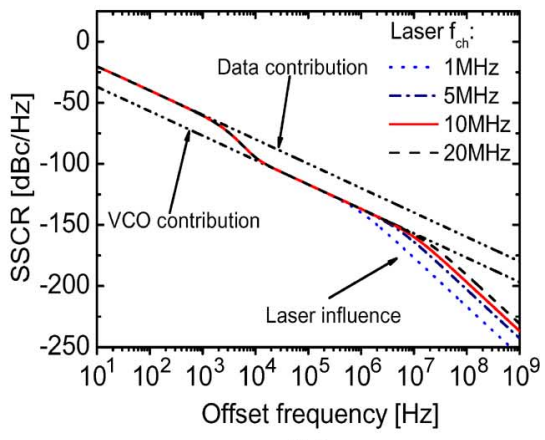

(b)

Fig. 5. SSCR of the recovered optical clock signal for selected values of the laser's characteristic knee frequency $f_{\text {ch. }}$. Input signal jitter: 160 fs. (a) Laser jitter: $100 \mathrm{fs}$ and VCO jitter: $\approx 0$ fs. (b) Laser jitter $\approx 0$ fs and VCO jitter $100 \mathrm{fs}$.

VCO signal timing jitter (jitter transfer function). In Fig. 4, the timing jitter of the extracted clock signal is plotted as a function of input OTDM data signal jitter for selected values of VCO jitter (225 fs, $711 \mathrm{fs}$, and $5 \mathrm{ps}$ ). The computations are made for the cases of zero and 140-ns time delay.

For the relatively large value of VCO jitter ( $5 \mathrm{ps}$ ), a large increase in clock jitter is observed as the time delay is increased from zero to 140 ns. Furthermore, it should be observed that the clock timing jitter is almost constant over a large range of input jitter values. The timing jitter of the VCO is large and therefore dominates the clock jitter. Reducing the timing jitter of the VCO reduces the impact of the time delay on the clock jitter, as seen in Fig. 4. For a relatively low VCO timing jitter of $225 \mathrm{fs}$, the impact of time delay on the clock jitter becomes negligible for input data signal jitter above approximately $100 \mathrm{fs}$. Furthermore, reducing the VCO jitter, the extracted clock jitter becomes more dependent on the input OTDM data signal jitter. The clock jitter approximately assumes the values of the input OTDM data signal jitter when the VCO jitter is $225 \mathrm{fs}$, and the time delay is zero.

So far, we have assumed that the VCO signal contained more phase noise that the input data signal. Now, let us consider a case in which the input signal is more noisy than the VCO signal. This is shown in Fig. 4(b), for selected values of the PI filter bandwidth: $100 \mathrm{kHz}, 5 \mathrm{MHz}$, and $15 \mathrm{MHz}$. As explained earlier, within the bandwidth of the PLL, the clock signal follows the input data signal, and for frequencies exceeding the PLL bandwidth, the extracted clock signal couples to the SSCR of the VCO signal. Since the free-running VCO signal contains less timing jitter than the input signal, the recovered optical clock signal will thus exhibit lower timing jitter than the input data signal. In the considered case, we want to decrease the PI filter bandwidth since the coupling frequency to the SSCR of the VCO signal decreases, as observed from Fig. 4(b), yielding an overall lower SSCR. However, even though the free-running VCO is less noisy than the input data signal, we may end up with a case where high-frequency noise is added to the extracted clock signal due to other noisy loop components. We may, however, conclude that the minimum requirement in order for a clock signal to exhibit less timing jitter than the input signal is to have a VCO, which contains less jitter than the input signal, and to have a low PLL bandwidth.

\section{Phase Noise Contributions From the Laser and the VCO}

In this section, we investigate how the SSCR of the recovered optical clock signal (at the laser output) is affected by the laser phase noise and the phase noise associated with the VCO. The input data is assumed to have higher jitter than the local oscillators (VCO and laser). The PLL bandwidth is chosen to be low in Fig. $5(20 \mathrm{kHz})$ in order to illustrate the coupling from data SSCR to the VCO and the laser SSCR.

In Fig. 5(a), the SSCR of the recovered $10-\mathrm{GHz}$ optical clock signal is shown. The laser characteristic knee frequency $f_{\text {ch }}$ is increased from 1 to $20 \mathrm{MHz}$. The phase noise of the free-running VCO is negligible, and the timing jitter of the free-running laser is $100 \mathrm{fs}$. Fig. 5(a) shows that within the PLL bandwidth $f_{\mathrm{pll}}$, the SSCR of the recovered optical clock 


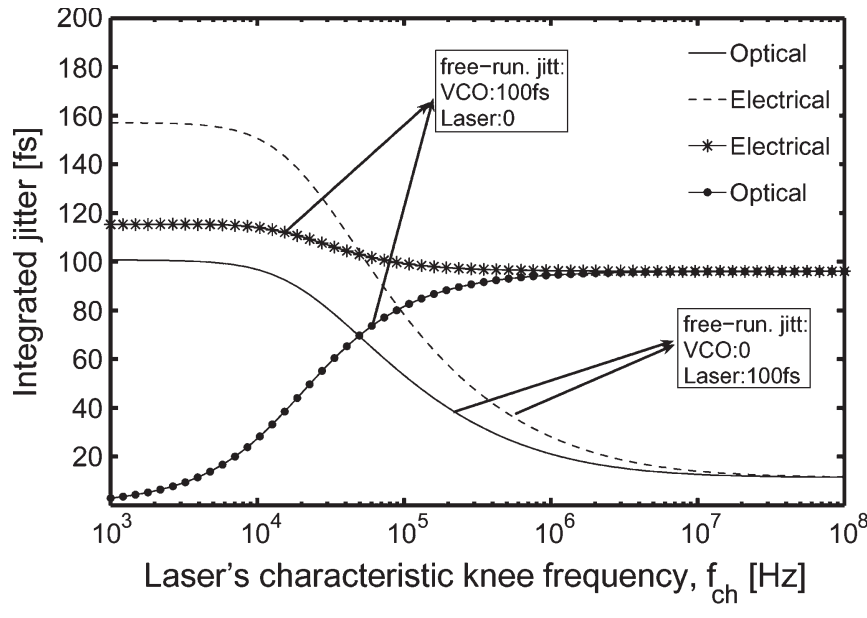

Fig. 6. Integrated timing jitter of the recovered optical and electrical clock signal as a function of the laser's characteristic knee frequency $f_{\mathrm{ch}}$. Input jitter: $160 \mathrm{fs}$. PLL bandwidth: $5 \mathrm{MHz}$. Jitter integration range: $20 \mathrm{kHz} f_{0} / 2$.

signal follows the SSCR of the input data signal. The laser characteristic knee frequency $f_{\text {ch }}$ determines the frequency at which the SSCR of the optical clock couples to the SSCR of the free-running laser. The SSCR of the optical clock signal is dominated by the VCO phase noise in the frequency range $f_{\text {pll }}-f_{\text {ch }}$. As $f_{\text {ch }}$ is increased, the SSCR of the optical clock signal is coupled to the VCO in a larger bandwidth, resulting in a bigger dip. In Fig. 5(b), we have assumed that the phase noise associated with the laser is negligible and the free-running VCO has a timing jitter of $100 \mathrm{fs}$. It is observed that as $f_{\text {ch }}$ is decreased, the SSCR is suppressed. By keeping $f_{\text {ch }}$ low, the VCO jitter is not transferred to the noise-free laser signal. Furthermore, the high-frequency jitter contribution $(>100 \mathrm{MHz})$ is reduced from the recovered optical clock signal, as observed in Fig. 5(b). It is therefore of great advantage to use a laser with low phase noise.

In Fig. 6, the integrated timing jitter of the recovered optical (laser output) and electrical (VCO output) clock signal is computed as a function of $f_{\mathrm{ch}}$. The PLL bandwidth is set to the more realistic value of $5 \mathrm{MHz}$, allowing for a reasonable locking time [35]. When the jitter of the free-running laser is $100 \mathrm{fs}$ and the corresponding jitter of the $\mathrm{VCO}$ is set to be negligible, the timing jitter of the recovered optical clock signal reduces as $f_{\text {ch }}$ increases. This is in accordance with Fig. 5(a). When the jitter associated with the free-running laser is negligible and the VCO free-running jitter is $100 \mathrm{fs}$, it is observed that the timing jitter of the recovered optical clock signal increases as $f_{\text {ch }}$ increases, in accordance with Fig. 5(b). For the extracted electrical clock signal, in both cases, the integrated timing jitter reduces as the laser's characteristic knee frequency increases. Furthermore, the recovered optical clock signal exhibits less timing jitter than the electrical clock signal in the range $1 \mathrm{kHz}-2 \mathrm{MHz}$.

\section{Reduction of Timing Jitter}

Optical re-amplification, re-shaping, and re-timing (3R) regeneration is potentially a key technology for bit rates of $40 \mathrm{~Gb} / \mathrm{s}$ and above. For $3 \mathrm{R}$ regeneration, clock signal extraction from a distorted data signal is needed, and the extracted clock

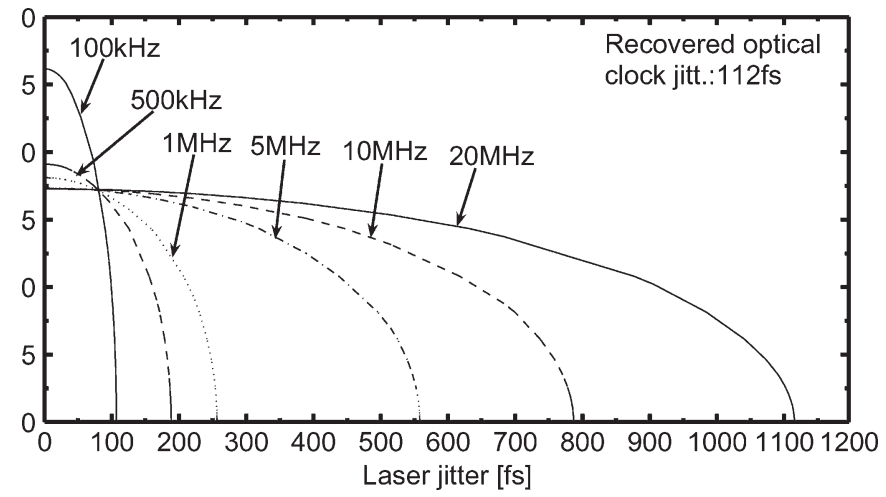

Fig. 7. Contour lines for a constant timing jitter of 112 fs of the recovered optical clock signal as a function of the VCO's and laser's free-running jitter when $f_{\mathrm{ch}}$ is varied in the range $100 \mathrm{kHz}-20 \mathrm{MHz}$. Input jitter: $160 \mathrm{fs}$. PLL bandwidth: $5 \mathrm{MHz}$. Jitter integration range: $20 \mathrm{kHz} f_{0} / 2$.

signal must exhibit lower timing jitter than the degraded data signal. CR has been performed by various techniques, e.g., by a self-pulsating DFB laser, a mode-locked laser, and an optical PLL; see, e.g., [36]. In order for a recovered clock signal to have less jitter than the input signal, the jitter of the free-running local oscillator must be less than that of the input signal [38]. An advantage of using an OPLL-based CR is that one has access to both a recovered electrical and optical clock signal. In this section, we describe the requirements to an OPLL to achieve reduced timing jitter.

Fig. 7 contour lines correspond to a constant timing jitter of 112 fs ( $\sim 30 \%$ jitter reduction compared to the input) of the recovered optical clock signal as a function of the free-running jitter of the VCO and the laser when the laser's characteristic knee frequency $f_{\text {ch }}$ is varied from $100 \mathrm{kHz}$ to $20 \mathrm{MHz}$. The input data signal jitter is 160 fs. In short, Fig. 7 shows the requirements for the free-running jitter of the laser and the VCO in order to obtain recovered optical clock signal with $30 \%$ jitter reduction compared to the input for a specific $f_{\mathrm{ch}}$.

Fig. 7 furthermore illustrates that as $f_{\text {ch }}$ is increased, the timing jitter requirement for the laser becomes more relaxed as the integrated clock signal jitter becomes less affected by the laser jitter. The VCO jitter requirements become more stringent as the laser's characteristic knee frequency $f_{\mathrm{ch}}$ is increased. However, this is insignificant when $f_{\mathrm{ch}}$ is above $500 \mathrm{kHz}$. It should be observed that even though the laser's free-running jitter exceeds $160 \mathrm{fs}$, an optical clock signal with lower timing jitter than at the input is obtainable if the jitter of the freerunning VCO is low enough.

Fig. 8 depicts level curves for a constant timing jitter of $112 \mathrm{fs}$ of the recovered electrical clock signal as a function of the free-running jitter to which the VCO and the laser again corresponds. Fig. 8 shows again the requirements for the freerunning jitter of the laser and the VCO in order to obtain recovered electrical clock signal with $30 \%$ jitter reduction compared to the input for a specific $f_{\text {ch }}$. Fig. 8 illustrates that as $f_{\text {ch }}$ is increased, the timing jitter requirement for the laser and for the VCO become more relaxed. By comparing Figs. 7 and 8, it is observed that the timing jitter requirements for the laser and the VCO are more stringent for the extracted electrical clock signal. 


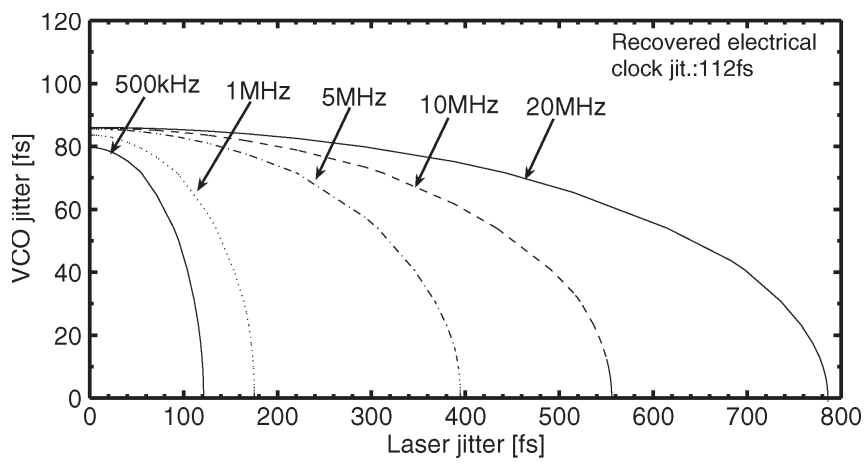

Fig. 8. Contour lines for a constant timing jitter of $112 \mathrm{fs}$ of the recovered electrical clock signal as a function of the VCO's and laser's free-running jitter when $f_{\mathrm{ch}}$ is varied in the range $500 \mathrm{kHz}-20 \mathrm{MHz}$. Input jitter: $160 \mathrm{fs}$. PLL bandwidth: $5 \mathrm{MHz}$. Jitter integration range: $20 \mathrm{kHz} f_{0} / 2$.

\section{CONCLUSION}

A detailed noise analysis of a CR circuit based on an OPLL, taking into account the phase noise associated with the signals and the effects of loop time delay and laser transfer function is presented. By a small-signal analysis, the Langevin equations describing the corresponding system are linearized to an Ornstein-Uhlenbeck process, and the correlation functions are obtained by the inverse Fourier transformation technique. Furthermore, the pdf of the recovered clock signal is computed. The noise analysis has shown that the minimum recovered clock signal jitter approaches the input data signal jitter if the input data signal has less jitter than the VCO and the laser. Increasing the loop length results in an increase in timing jitter of the recovered clock signal as the time delay approaches its critical value. The impact of time delay on the clock jitter can be reduced by using a low-noise VCO and a low PI filter bandwidth. Furthermore, we have numerically investigated the influence of noisy electrical and optical local oscillators on the timing jitter of the recovered clock signal. Timing jitter requirements for the laser can be significantly relaxed by a large laser characteristic knee frequency using a low-noise VCO. If the laser has more jitter than the VCO, it is preferable to have a large laser characteristic knee frequency, and if the laser has less jitter than the VCO, it is preferable to have a small laser characteristic knee frequency. We have also shown that the recovered optical clock signal exhibits lower timing jitter than the recovered electrical clock signal for low values of the laser characteristic knee frequency.

\section{APPENDIX}

In this section, it is shown how timing jitter (temporal accuracy) of a signal having Brownian motion phase error can be computed. We start by considering the intensity of an ideal periodic pulse train expressed in a Fourier series:

$$
g(t)=a_{0}+\sum_{i=1}^{\infty} a_{i} \sin \left[2 \pi i f_{0} t\right]
$$

where $a_{i}$ are Fourier coefficients, $f_{0}$ is the repetition frequency, and $i$ is an integer. The effect of phase noise $\alpha(t)$ on a signal $g(t)$ creates deviations or jitter in the repetition frequency. In other words, phase noise $\alpha(t)$ will cause spectral dispersion of a signal. We are going to model $\alpha(t)$ as a Brownian motion phase error [25]. The signal $g(t)$ becomes $g(t+\alpha(t))$ in the presence of the phase noise and is expressed as

$$
g(t+\alpha(t))=a_{0}+\sum_{i=1}^{\infty} a_{i} \sin \left[2 \pi i f_{0} t+2 \pi i f_{0} \alpha(t)\right] .
$$

A randomly fluctuating phase noise $\alpha(t)$ can be asymptotically described as a modulated Wiener process [25]:

$$
\frac{d \alpha}{d t}=\sqrt{c} \Gamma(t)
$$

where $c$ is a constant determining the amount of phase noise associated with the signal. The constant $c$ will depend on the various parameters of the optical communication system, i.e., pulse source at the transmitter, noise figure of the channel and receiver, etc., where $\Gamma(t)$ is a stochastic Langevin noise force which is Gaussian distributed and is fully characterized by its mean value and correlation function:

$$
\langle\Gamma(t)\rangle=0 \quad\left\langle\Gamma(t) \Gamma\left(t^{\prime}\right)\right\rangle=\delta\left(t-t^{\prime}\right) .
$$

Using (67) and (68), the phase noise can be statistically characterized [33]:

$$
\langle\alpha(t)\rangle=0 \quad\left\langle\left|\alpha(t)-\alpha\left(t^{\prime}\right)\right|^{2}\right\rangle=c\left|t-t^{\prime}\right| .
$$

The ensemble average of the phase noise is thereby zero, and its mean-square value increases linearly with time. For practical applications, we are interested in obtaining the power frequency spectrum of the signal $g(t+\alpha(t))$. By integrating the frequency spectrum, we can then compute the timing jitter of the signal. The single-sided frequency spectrum of a signal $g(t+\alpha(t))$ is expressed as [25]

$$
G(f)=\sum_{i=-\infty}^{\infty} \frac{\left|a_{i}\right|^{2} f_{0}^{2} i^{2} c^{2}}{\pi^{2} f_{0}^{4} i^{2} c^{2}+\left(f+i f_{0}\right)^{2}} .
$$

Using the frequency spectrum of the signal in (70), the timing jitter (around the first harmonic) can then be calculated using the von der Linde method [39]:

$$
\tau_{\mathrm{jitt}}=\frac{1}{2 \pi f_{0}} \sqrt{2 \int_{f_{\min }}^{f_{\max }} \frac{G\left(f_{0}+f\right)}{\left|a_{1}\right|^{2}} d f}
$$

where $f_{\min }$ and $f_{\max }$ are lower and upper integration limits, respectively. Inserting $G\left(f_{0}+f\right)$ in (71) and performing the integration around the first harmonic $(i=1,-1)$, the timing jitter of a signal having Brownian motion phase error becomes

$$
\begin{aligned}
\tau_{\mathrm{jitt}}= & \frac{1}{\sqrt{2} \pi \sqrt{\pi} f_{0}} \\
& \cdot\left[\arctan \left(\frac{f_{\mathrm{max}}}{\pi f_{0}^{2} c}\right)+\arctan \left(\frac{f_{\max }+2 f_{0}}{\pi f_{0}^{2} c}\right)\right. \\
& \left.-\arctan \left(\frac{f_{\mathrm{min}}}{\pi f_{0}^{2} c}\right)-\arctan \left(\frac{f_{\min }+2 f_{0}}{\pi f_{0}^{2} c}\right)\right]^{1 / 2} .
\end{aligned}
$$


Using (72), we can compute timing jitter of a signal as a function of constant $c$ for the specified integration range $f=\left[f_{\min } ; f_{\max }\right]$.

\section{ACKNOWLEDGMENT}

The authors would like to thank P. Jeppesen and B. Tromborg for technical discussions and guidance.

\section{REFERENCES}

[1] W. C. Lindsey, Synchronization Systems in Communication and Control. Englewood Cliffs, NJ: Prentice-Hall, 1972.

[2] F. M. Gardner, Phaselock Teschnique. New York: Wiley, 2005.

[3] R. E. Best, Phase-Locked Loops. New York: McGraw-Hill, 1984.

[4] J. Klapper and J. T. Frankle, Phase-Locked and Frequency-Feedback Systems. New York: Academic, 1972.

[5] D. O. Pederson and K. Mayaram, Analog Integrated Circuits for Communication. Boston, MA: Kluwer, 1991, ch. 12-14.

[6] M. Mansuri and C. K. Yang, "Jitter optimization on phase-locked loop design parameters," IEEE J. Solid-State Circuits, vol. 37, no. 11, pp. 1375-1382, Nov. 2002.

[7] A. Mehrotra, "Noise analysis of phase-locked loops," IEEE Trans. Circuits Syst. I, Fundam. Theory Appl., vol. 49, no. 9, pp. 1309-1316, Sep. 2002.

[8] B. Razavi, Monolithic Phase-Locked Loops and Clock Recovery Circuits. Piscataway, NJ: IEEE Press, 1992.

[9] K. Osamu and K. Satoki, "Prescaled timing extraction from $400 \mathrm{~Gb} / \mathrm{s}$ optical signal using a phase lock loop based on four-wave-mixing in a laser diode amplifier," J. Lightw. Technol., vol. 8, no. 8, pp. 1094-1096, Aug. 1996.

[10] _ "Ultrahigh-speed clock recovery circuit based on four wave mixing in a traveling-wave diode amplifier," J. Lightw. Technol., vol. 11, no. 12, pp. 1757-1767, Aug. 1996.

[11] D. Tong, B. Mikkelsen, G. Raybon, T. N. Nielsen, F. K. Dreyer, and J. E. Johnson, "Optoelectronic phase-locked loop with balanced photodetection for clock recovery in high-speed optical time-divisionmultiplexing systems," IEEE Photon. Technol. Lett., vol. 12, no. 8, pp. 1064-1066, Aug. 2000.

[12] L. Oxenløwe, C. Schubert, C. Shmidt, E. Hilliger, J. Berger, U. Feiste, R. Ludwig, and H. G. Weber, "Optical clock recovery employing an optical PLL using cross-phase modulation in a Sagnac-interferometer," presented at the IEEE Conf. Lasers and Electro-Optics (CLEO), Baltimore, MD, 2001.

[13] S. Kawanishi, H. Takara, K. Uchiyama, I. Shake, and K. Mori, "3 Tb/s (160 Gb/s/spl times/19 ch) OTDM/WDM transmission experiment," presented at the IEEE Optical Fiber Commun. Conf. (OFC), Nice, France, 1999, Paper ThA3.

[14] S. Kawanishi, H. Takara, M. Saruwatari, and N. Kioth, "Ultra-high speed phasedlocked-loop-type clock recovery circuit using a traveling-wave laser diode amplifier as a $50 \mathrm{GHz}$ phase detector," Electron. Lett., vol. 29, no. 19 , pp. $769-771$, Sep. 1995 .

[15] T. Yamamoto, U. Feiste, J. Berger, C. Schubert, C. Schmidt, R. Ludwig, and H. G. Weber, " $160 \mathrm{~Gb} / \mathrm{s}$ demultiplexer with clock recovery using SOA-based interferometric switches and its application to $120 \mathrm{~km}$ fiber transmission," presented at the Eur. Conf. Optical Commun. (ECOC), Amsterdam, The Netherlands, 2001, Paper Tu.L.2.6.

[16] L. K. Oxenløwe, A. I. Siahlo, K. S. Berg, A. T. Clausen, B. M. Sørensen, K. Yvind, and P. Jeppesen, "A novel $160 \mathrm{~Gb} / \mathrm{s}$ receiver configuration including a glass crystal pulsed laser, photonoc crystal fiber and a simple clock recovery scheme," presented at the Eur. Conf. Optical Commun. (ECOC), Rimini, Italy, 2003, Paper Th2.5.3.

[17] E. Hashimoto, A. Takada, and Y. Katagiri, "High-frequency synchronized signal generation using semiconductor lasers," IEEE Trans. Microw. Theory Tech., vol. 47, no. 7, pp. 1206-1218, Jul. 1999.

[18] C. M. DePriest, T. Yilmaz, A. Braun, J. Abeles, and P. J. Delfyett, "High-quality photonic sampling stream from a semiconductor diode ring laser," IEEE J. Quantum Electron., vol. 38, no. 4, pp. 380-389, Apr. 2002

[19] D. J. Jones, K. W. Holman, M. Notcutt, J. Ye, J. Chandalia, L. A. Jiang, E. Ippen, and H. Yokoyama, "Ultralow-jitter, 1550-nm mode-locked semiconductor laser synchronized to a visible opti- cal frequency standard," Opt. Lett., vol. 28, no. 10, pp. 813-815, May 2003.

[20] K.-P. Ho and J. M. Kahn, "Electronic compensation to mitigate nonlinear phase noise," J. Lightw. Technol., vol. 22, no. 3, pp. 779-783, Mar. 2004.

[21] M. A. Grant, W. C. Michie, and M. J. Fletcher, "The performance of optical phase-locked loops in the presence of nonnegligible loop propagation delay," J. Lightw. Technol., vol. LT-5, no. 4, pp. 592-597, Apr. 1987.

[22] D. Zibar, L. K. Oxenløwe, A. T. Clausen, J. Mørk, and P. Jeppesen, "Analysis of the effects of time delay in clock recovery circuits based on phase-locked loops," presented at the IEEE Laser Electro Optics Society (LEOS), Rio Grande, Puerto Rico, 2004, Paper TuR4.

[23] C. W. Gardiner, Handbook of Stochastic Methods, Springer-Verlag, Berlin, Germany, 1983.

[24] M. Nakazawa, T. Yamamoto, and K. R. Tamura, "1.28 Tb/s-70 km OTDM transmission using third and fourth order simultaneous dispersion compensation with a phase modulator," Electron. Lett., vol. 36, no. 24 , pp. 2027-2029, Nov. 2000.

[25] A. Demir, "Analysis and simulations of noise in nonlinear electronic circuits and systems," Ph.D. dissertation, Univ. California, Berkeley, CA, 1997.

[26] A. Demir, A. Mehrotra, and J. Roychowdhury, "Phase noise in oscillators: A unifying theory and numerical methods for characterization," IEEE Trans. Circuits Syst. I, Fundam. Theory Appl., vol. 47, no. 5, pp. 655-674, May 2000.

[27] K. Yvind, "Semiconductor mode-locked lasers for optical communication systems," Ph.D. dissertation, Res. Centre COM, Tech. Univ. Denmark, Lyngby, Denmark, 2003.

[28] D. Larsson, K. Yvind, and J. M. Hvam, "Wide-band residual phasenoise measurements on $40-\mathrm{GHz}$ monolithic mode-locked lasers," IEEE Photon. Technol. Lett., vol. 17, no. 11, pp. 2388-2390, Nov. 2005

[29] A. M. Braun, V. B. Khalfin, M. H. Kwakernaak, W. F. Reichert, L. A. DiMarco, Z. A. Schellenberger, C. M. DePriest, T. Yilmaz, P. J. Delfyett, and J. H. Abeles, "Universality of model-locked jitter performance," IEEE Photon. Technol. Lett., vol. 14, no. 8, pp. 1058-1060, Aug. 2002.

[30] A. T. Clausen, H. N. Poulsen, L. K. Oxenløwe, A. Siahlo, J. Seone, and P. Jeppesen, "Pulse Source Requirements for OTDM Systems," presented at the Laser Electro Optics Society (LEOS) Conf., Tucson, AZ, 2003, Paper TyY2.

[31] J. Buckwalter and Y. A. Robert, "Time delay considerations in highfrequency phase-locked loops," presented at the Radio Frequency Integrated Circuits Symp., Seattle, WA, 2002, Paper MO3D-5.

[32] A. Papoulis, Probability, Random Variables and Stochastic Processes, 3rd ed. New York: McGraw-Hill, 1991.

[33] H. Risken, The Fokker-Planck Equation, 2nd ed. New York: SpringerVerlag, 1989.

[34] L. K. Oxenløwe, A. I. Siahlo, K. S. Berg, A. T. Clausen, K. Yvind, B. M. Sørensen, and P. Jeppesen, "Pre-scaled clock recovery with compact semiconductor devices for ultra high-speed OTDM systems," presented at the IEEE Eur. Conf. Optical Commun. (ECOC), Stockholm, Sweden, 2004, Paper We3.5.2.

[35] D. Zibar, L. K. Oxenløwe, A. T. Clausen, and J. Mørk, "Theoretical and experimental investigation of a balanced phase-locked loop based clock recovery at a bit rate of $160 \mathrm{~Gb} / \mathrm{s}$," presented at the IEEE Laser Electro Optics Soc. (LEOS) Conf., Tucson, AZ, 2003, Paper TuY5.

[36] J. Lasri, P. Devgan, R. Tong, and P. Kumar, "Ultralow timing jitter $40 \mathrm{~Gb} / \mathrm{s}$ clock recovery using self-starting optoelectronic oscillator," IEEE Photon. Technol. Lett., vol. 16, no. 1, pp. 263-265, Jan. 2004.

[37] L. K. Oxenløwe, L. J. Christiansen, D. Larsson, K. Yvind, A. T. Clausen, J. Seone, A. Siahlo, B. M. Sørensen, and P. Jeppesen, "Lowjitter prescaled clock recovery with compact semiconductor components for ultra high-speed OTDM systems," in Proc. IEEE OECC, Yokohama, Japan, 2004, pp. 888-889.

[38] D. Zibar, L. K. Oxenløwe, J. Mørk, M. P. Sørensen, M. Galili, A. T. Clausen, B. Sørensen, and P. Jeppesen, "Detailed modelling and experimental characterisation of an ultra-fast optoelectronic clock recovery circuit," presented at the IEEE Eur. Conf. Optical Commun. (ECOC), Glasgow, U.K., 2005, Paper We4.P.111.

[39] D. von der Linde, "Characterization of the noise in continuously operating mode-locked laser," Appl. Phys. B, Photophys. Laser Chem., vol. 4, no. 201 , pp. 201-217, 1986. B(39). 


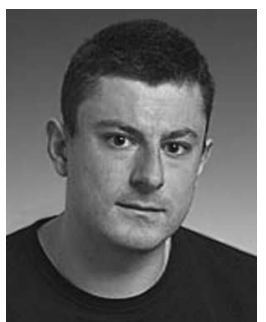

Darko Zibar (S'02-M'04) was born in Belgrade, former Yugoslavia, on December 9, 1978. He received the M.Sc. degree in telecommunication from the Technical University of Denmark, Lyngby, Denmark, in 2004. He is currently working toward the Ph.D. degree at the Department of Communications, Optics and Materials, COM-DTU within the field of short pulse sources for high-speed functionalities. The work is part of the National Danish project Ultra-Net funded by the Danish research council.

He was a Visiting Researcher with Optoelectronic Research Group led by Prof. J. E. Bowers at the University of California, Santa Barbara, from January 2006 to August 2006, working on coherent receivers for phase-modulated analog optical links. His research interests are in the area of optical communication, with emphasis on stochastic modeling and numerical methods.

Mr. Zibar is a recipient of the Best Student Paper Award at the IEEE Microwave Photonics Conference in 2006 for his work on a novel optical phase demodulator based on a sampling phase-locked loop.

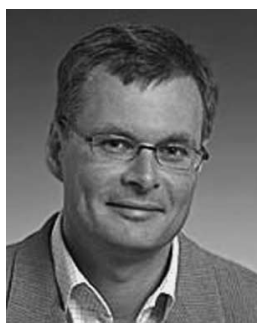

Jesper Mørk received the M.Sc., Ph.D., and Dr. Techn. degrees from the Technical University of Denmark (DTU), Lyngby, Denmark, in 1986, 1988, and 2003, respectively.

Since 2002, he has been Professor of semiconductor devices for optical communication systems and is the Deputy Head of the nanophotonics area at the Department of Communications, Optics and Materials, DTU. His current research interests are in the area of device physics, in particular ultrafast devices for optical signal processing, noise in nonlinear devices, and quantum photonics.

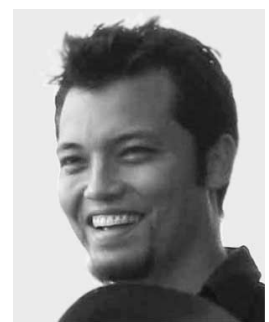

Leif Katsuo Oxenløwe received the B.Sc. degree in physics and astronomy from Niels Bohr Institute, University of Copenhagen, Copenhagen, Denmark, in 1996, the International Diploma from the Imperial College of Science, Technology, and Medicine, London, U.K., and the M.Sc. degree from the University of Copenhagen in 1998, and the Ph.D. degree from the Technical University of Denmark, Lyngby, Denmark, in 2002.

$\mathrm{He}$ is currently an Assistant Professor in the Systems Competence Area at Research Center COM, Lyngby. He is working on experimental research in the field of ultrafast optical communications (at or above $160 \mathrm{~Gb} / \mathrm{s}$ ). He has been working within the $\mathrm{EU}$ IST project TOPRATE and has been involved in the Danish research council financed project SCOOP. Since May 2004, he has been with and managed the project Ultrahigh speed data rates for future generation Internet (Ultra-Net) funded by the Danish research council. The aim of the project is to explore techniques to regenerate and process data at $160 \mathrm{~Gb} / \mathrm{s}$ data rates and above.

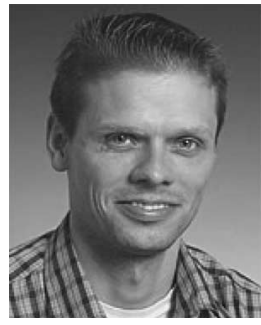

Anders T. Clausen received the M.Sc. degree from the Institute for Electromagnetic Systems, currently Research Center COM, Lyngby, Denmark, in 1997. $\mathrm{He}$ is currently working toward the Ph.D. degree at the Technical University of Denmark, Lyngby, focusing on optical communication systems with terabits per second potential in parallel with his work as Project Manager of COM contributions to the European IST project TOPRATE, where all aspects of ultrahigh speed - to be exact $160 \mathrm{~Gb} / \mathrm{s}$ and beyond-optical time division multiplexing (OTDM) systems are addressed, i.e., transmitter and receiver implementation combined with transmission aspects.

He is currently Head of the OTDM group, which is subgroup of the System Competence Area at the COM Center. After graduation, he continued at EMI/COM as a Research Associate as part of the team working on the European ACTS project HIGHWAY. Here, he was involved in implementation and research of OTDM systems operating at 40,80 , and $160 \mathrm{~Gb} / \mathrm{s}$, with special focus on demultiplexing, wavelength conversion, clock extraction, and dispersion compensation. After successful finalization of the HIGHWAY project, his work focused on regeneration of data signals in OTDM systems. This paper was carried out within the European project REPEAT. 